\title{
CfA4: LIGHT CURVES FOR 94 TYPE Ia SUPERNOVAE
}

\author{
Malcolm Hicken $^{1}$, Peter Challis ${ }^{1}$, Robert P. Kirshner ${ }^{1}$, Armin Rest $^{2}$, Claire E. Cramer $^{3}$, W. Michael Wood-Vasey ${ }^{4}$, \\ Gaspar Bakos $^{1,5}$, Perry Berlind ${ }^{1}$, Warren R. Brown ${ }^{1}$, Nelson Caldwell ${ }^{1}$, Mike Calkins ${ }^{1}$, Thayne Currie ${ }^{6}$, \\ Kathy de KleEr $^{7}$, Gil Esquerdo ${ }^{8}$, Mark Everett ${ }^{8}$, Emilio Falco ${ }^{1}$, Jose Fernandez ${ }^{1}$, Andrew S. Friedman ${ }^{1}$, \\ Ted Groner $^{1}$, Joel Hartman ${ }^{1,5}$, Matthew J. Holman ${ }^{1}$, Robert Hutchins ${ }^{1}$, Sonia Keys ${ }^{1}$, David Kipping ${ }^{1}$, Dave Latham ${ }^{1}$, \\ George H. Marion ${ }^{1}$, Gautham Narayan ${ }^{1}$, Michael Pahre ${ }^{1}$, Andras Pal ${ }^{1}$, Wayne Peters ${ }^{1}$, Gopakumar Perumpilly ${ }^{9}$, \\ Ben Ripman $^{1}$, Brigitta Sipocz ${ }^{1}$, Andrew Szentgyorgyi ${ }^{1}$, Sumin Tang ${ }^{1}$, Manuel A. P. Torres $^{1}$, Amali Vaz $^{10}$, \\ ScOTT WOLK ${ }^{1}$, AND ANDREAS ZeZAs ${ }^{1}$ \\ ${ }^{1}$ Harvard-Smithsonian Center for Astrophysics, Cambridge, MA 02138, USA; mhicken@ cfa.harvard.edu \\ ${ }^{2}$ Space Telescope Science Institute, Baltimore, MD 21218, USA \\ ${ }^{3}$ NIST (National Institute of Standards and Technology), Gaithersburg, MD 20899, USA \\ ${ }^{4}$ Department of Physics and Astronomy, University of Pittsburgh, Pittsburgh, PA 15260, USA \\ ${ }^{5}$ Department of Astrophysical Sciences, Princeton University, Princeton, NJ 08542, USA \\ ${ }^{6}$ NASA, Goddard Space Flight Center, Greenbelt, MD 20771, USA \\ ${ }^{7}$ Department of Physics, Massachusetts Institute of Technology, Cambridge, MA 02139, USA \\ ${ }_{8}$ Planetary Science Institute, 1700 East Fort Lowell Road, Tucson, AZ 85719, USA \\ ${ }^{9}$ Department of Physics, University of South Dakota, Vermillion, SD 57069, USA \\ ${ }^{10}$ Department of Physics, Harvard University, Cambridge, MA 02138, USA \\ Received 2012 February 14; accepted 2012 April 5; published 2012 May 17
}

\begin{abstract}
We present multi-band optical photometry of 94 spectroscopically confirmed Type Ia supernovae (SNe Ia) in the redshift range $0.0055-0.073$, obtained between 2006 and 2011. There are a total of 5522 light-curve points. We show that our natural-system SN photometry has a precision of $\lesssim 0.03$ mag in $B V r^{\prime} i^{\prime}, \lesssim 0.06$ mag in $u^{\prime}$, and $\lesssim 0.07$ mag in $U$ for points brighter than $17.5 \mathrm{mag}$ and estimate that it has a systematic uncertainty of $0.014,0.010,0.012,0.014$, 0.046 , and $0.073 \mathrm{mag}$ in $B V r^{\prime} i^{\prime} u^{\prime} U$, respectively. Comparisons of our standard-system photometry with published SN Ia light curves and comparison stars reveal mean agreement across samples in the range of $\sim 0.00-0.03 \mathrm{mag}$. We discuss the recent measurements of our telescope-plus-detector throughput by direct monochromatic illumination by Cramer et al. This technique measures the whole optical path through the telescope, auxiliary optics, filters, and detector under the same conditions used to make SN measurements. Extremely well characterized natural-system passbands (both in wavelength and over time) are crucial for the next generation of SN Ia photometry to reach the 0.01 mag accuracy level. The current sample of low- $z$ SNe Ia is now sufficiently large to remove most of the statistical sampling error from the dark-energy error budget. But pursuing the dark-energy systematic errors by determining highly accurate detector passbands, combining optical and near-infrared (NIR) photometry and spectra, using the nearby sample to illuminate the population properties of SNe Ia, and measuring the local departures from the Hubble flow will benefit from larger, carefully measured nearby samples.
\end{abstract}

Key word: supernovae: general

Online-only material: machine-readable tables

\section{INTRODUCTION}

The idea of using supernovae ( $\mathrm{SNe}$ ) as tools for measuring the properties of cosmic expansion has a long history (Kirshner 2010). Pioneering work by the Calan/Tololo survey produced the first large sample of SN Ia light curves, with 29 SN Ia light curves measured with CCD detectors (Hamuy et al. 1996b). Insight from Mark Phillips helped sharpen the use of SNe Ia for distance determinations (Phillips 1993; Hamuy et al. 1996a). At the Harvard-Smithsonian Center for Astrophysics (CfA), we have been engaged in building up the local sample, with 22 SN Ia light curves in the CfA1 sample (Riess et al. 1999), 44 in CfA2 (Jha et al. 2006), and 185 in CfA3 (Hicken et al. 2009a, hereafter H09). Additionally, Krisciunas and colleagues have published a significant number (Krisciunas et al. 2000, 2001, 2003, 2004b, 2004c, 2006), the European Supernova Collaboration has published photometry of various nearby SNe Ia (see Stanishev et al. 2007; Elias-Rosa et al. 2008; Taubenberger et al. 2008, and references therein), and Kowalski et al. (2008) published eight nearby SNe Ia. More recently, the Sloan Digital Sky Survey (SDSS) published 19 SNe Ia with $z<0.100$ (Holtzman et al. 2008) as part of the SDSS-II program, and the Lick Observatory Supernova Search (LOSS) published optical photometry of 165 SNe Ia (Ganeshalingam et al. 2010). The Carnegie Supernova Project (CSP) produced 35 SN Ia light curves in its first release (Contreras al. 2010, hereafter CSP1) and 50 in its second (Stritzinger et al. 2011, hereafter CSP2), with a majority of these objects including nearinfrared (NIR) photometry.

Looking to the future, CfA, LOSS, and CSP continue building further nearby samples. Of particular interest will be $\sim 100$ CfA NIR light curves that have optical photometry from CfA3 and CfA4 (Wood-Vasey et al. 2008; A. Friedman et al. 2012, in preparation). In conjunction with this NIR and optical photometry, the CfA Supernova Group has taken spectra of many of these SNe (Matheson et al. 2008; Blondin et al. 2011; Blondin et al. 2012) using the FAST spectrograph (Fabricant et al. 1998).

From its second and third years, SDSS-II should have photometry of another few dozen spectroscopically confirmed SNe Ia at $z<0.1$. The Palomar Transient Factory has discovered and spectroscopically confirmed $\sim 900 \mathrm{SNe}$ Ia in its first two 
years, ${ }^{11}$ and a large number of these should have quality light curves. The Nearby Supernova Factory (Aldering et al. 2002) has made spectrophotometric observations of several hundred SNe Ia. ESSENCE will shortly publish their whole data set and cosmological analysis (G. Narayan et al. 2012, in preparation). PanSTARRS ${ }^{12}$ is also producing large numbers of SN Ia light curves that span the range from low $-z$ to cosmologically telling redshifts with a single photometric system that should diminish the photometric uncertainties encountered by splicing together separate samples at low and high redshift.

In addition to measuring $\mathrm{SN}$ data, we have also been engaged in improving the methods for determining SN Ia distances, using observations in multiple photometric bands to estimate both the luminosity of an SN Ia and its extinction (Riess et al. 1996; Jha et al. 2007). MLCS2k2 is the most recent incarnation. SALT2 (Guy et al. 2007) and SiFTO (Conley et al. 2008) are other popular light-curve fitters. The current state of the art uses a statistical model for light-curve shapes that predicts distances to $7 \%$ for well-observed SNe Ia using optical data and 5\% when NIR photometry is added (Mandel et al. 2011).

The application of the published nearby samples to cosmology includes the determination of $H_{o}$ to the $3 \%$ level from the intercept of the Hubble diagram (Riess et al. 2011), where over half of the SN Ia sample came from CfA3. In addition, the photometry for two of the eight Cepheid-calibrated SNe Ia (SN 2007af and SN 2007sr) came from the CfA3 sample, and the improved photometric calibration of the comparison stars of a third (SN 1995al) was obtained from observations taken at the F. L. Whipple Observatory (FLWO) $1.2 \mathrm{~m}$ telescope during the course of the CfA3 observations (Riess et al. 2009). Measurement of $q_{o}$ has been a major application that requires both high- and low-redshift samples that are on a common photometric system and analyzed in a consistent way. The pioneering work of measuring distant SNe Ia, first published by Riess et al. (1998) and subsequently by Perlmutter et al. (1999), led to the surprising result of cosmic acceleration: $q_{o}<0$. Until 2009, the paucity of low-redshift $\mathrm{SNe}$ Ia was a significant contributor to the statistical uncertainty in dark-energy properties. This changed in 2009, when Hicken et al. (2009b) used the CfA3 data and data from the literature to construct the Constitution sample of SN Ia redshifts and distances and employed it to improve constraints on the dark-energy equation-of-state parameter, $w$. The CfA3 sample was also instrumental in providing the data for Kelly et al. (2010), who first detected the small but real relation between $\mathrm{SN}$ Ia host-galaxy masses and the residuals from the distance predictions based on SN Ia light curves. This was confirmed at higher redshift by work based on the Sloan SN survey (Lampeitl et al. 2010) and on the Supernova Legacy Survey (Sullivan et al. 2010). A constraint on $\sigma_{8}$, the amplitude of cosmic fluctuations, based in large part on the CfA3 sample, has recently been derived by Turnbull et al. (2012), who used the velocity residuals in the nearby Hubble flow to determine the variance in the dark matter density on a spatial scale of 8 Mpc, $\Omega_{m}^{0.55} \sigma_{8, \text { lin }}=0.40 \pm 0.07$.

Amanullah et al. (2010) presented the Union 2.0 set of SN Ia distances, incorporating the CfA3 and first-year SDSS-II phototomery (Holtzman et al. 2008), while Suzuki et al. (2012) added 16 cosmologically useful SNe Ia from the Hubble Space Telescope Cluster Supernova Survey, with 10 at $z>1$, to form the Union 2.1 set and provide tight limits on constant $w$ in a flat

\footnotetext{
11 http://www.astro.caltech.edu/ptf/

12 http://pan-starrs.ifa.hawaii.edu/public/science-goals/active-universe.html
}

CDM model: $1+w=-0.013_{-0.073}^{+0.068}$, where the uncertainties include all statistical and systematic errors. Sullivan et al. (2011) combine all three years of the Supernova Legacy Survey data with the nearby, first-year SDSS-II and Higher- $z$ (Riess et al. 2007) samples to measure $1+w=0.061_{-0.068}^{+0.069}$, where a flat universe is assumed and the uncertainties include all statistical and SN Ia systematic errors. The Union 2.1 and Supernova Legacy Survey measurements of $w$ are the state of the art at the moment.

Since systematic errors in dark-energy properties are now equal to or larger than the errors produced by finite sample sizes, progress demands ways to decrease those systematic errors. Two of the largest sources of systematic uncertainty in using $\mathrm{SNe}$ Ia for cosmology are (1) the entanglement of intrinsic SN Ia color and host-galaxy reddening and (2) the overall accuracy of SN Ia photometry, especially in accurately characterizing the passbands used.

One promising path to overcoming the first source of systematic error is through NIR observations of SNe Ia. As shown by Krisciunas et al. (2004a), Wood-Vasey et al. (2008), CSP1, CSP2, and Mandel et al. (2011), SNe Ia are better standard candles in the NIR and extinction by dust is a less vexing problem. The combination of the optical and NIR photometry from the CSP and the CfA will lead to improved disentangling of SN Ia color and host reddening. Another interesting path to better distances and better understanding of the nature of SNe Ia comes through combining information from light curves with information from spectra. Recent work by Blondin et al. (2011) shows that spectra can be used to determine the intrinsic luminosity of SNe Ia from the CfA samples. This builds upon findings by Nugent et al. (1995), Bongard et al. (2006), Hachinger et al. (2008), and Bailey et al. (2009). Silverman et al. (2012) combine nearby SN Ia spectra and photometry to achieve the largest reduction in the Hubble diagram scatter via adding spectra to date. Unlike the broad cosmological problem, the newer areas of combining NIR photometry and optical spectra with optical photometry have significantly less data. This paper, with a sample of 94 new optical light curves, has substantial overlap with CfA NIR photometry (A. Friedman et al. 2012, in preparation) and optical spectra (Blondin et al. 2012) of the same objects. We expect that these measurements will be valuable in developing the tools to improve our knowledge of SNe Ia and of the expansion history of the universe.

The second large source of systematic uncertainty will be greatly reduced through better characterization of detector passbands, as was done for our measurements by C. Cramer et al. (2012, in preparation) using a monochromatic source to determine the system throughput, as described below. In addition to better passband characterization, the significant overlap of nearby SN Ia photometry in the published CfA, LOSS, CSP, and other samples will aid in better understanding any possible systematic photometric effects that a given sample might have.

In Section 2, we describe our observational and data reduction procedures. Greater emphasis is placed on the few differences as compared with the CfA3 procedures, and a briefer description is provided where the procedures remained the same. We then present the CfA4 light curves. In Section 3, we compare the overlapping objects between CfA4, LOSS, and CSP. The CfA4 light curves, comparison star magnitudes, and passbands can be found at our Web site ${ }^{13}$ or in the online version of this paper.

\footnotetext{
13 http://www.cfa.harvard.edu/supernova/CfA4
} 


\section{DATA AND REDUCTION}

The CfA4 sample consists of 5522 light-curve points. All 94 SNe have $B V r^{\prime} i^{\prime}$ measurements, while 14 have $U$ and 12 have $u^{\prime}$. The average number of light-curve points per $\mathrm{SN}$ is 14.9 in $V r^{\prime} i^{\prime}, 12.5$ in $B, 7.8$ in $u^{\prime}$, and 7.0 in $U$. The closest redshift, $z_{\mathrm{CMB}}$, is 0.0055 , and the farthest is 0.073 . The median redshift is 0.029 , while the 25 th and 75 th percentile redshifts are 0.017 and 0.038 , respectively. Eighty-nine of $94 \mathrm{SNe}$ have $z_{\mathrm{CMB}}>0.010$.

CfA4 data processing followed the same three steps used for CfA3: reduction, calibration, and host-galaxy subtraction. Here we provide a brief overview of the overall process (see H09 for a more detailed treatment) and describe the differences between CfA4 and CfA3 in greater depth. The reduction and subtraction stages were carried out by a version of the ESSENCE and SuperMACHO pipeline (Miknaitis et al. 2007; Rest et al. 2005; Garg et al. 2007). Calibration paralleled CfA3 but was more automated. We employed differential photometry, calibrating the comparison stars surrounding the $\mathrm{SN}$ on photometric nights and then measuring the flux of the $\mathrm{SN}$ relative to the comparison stars in each data image, on both photometric and non-photometric nights. We employed host-galaxy subtraction for all $94 \mathrm{SNe}$, using multiple reference images for the majority of the SN.

\subsection{Instruments}

The CfA4 data were obtained on the $1.2 \mathrm{~m}$ telescope at the FLWO using the single-chip, four-amplifier CCD KeplerCam. ${ }^{14}$ Observations were acquired on amplifier two with a pixel scale of $0{ }^{\prime} 672$, resulting in a field of view of approximately $11.5 \times 11.5$. The $1.2 \mathrm{~m}$ primary mirror deteriorated during the course of the CfA4 observing, and its effects will be described below. A replacement mirror is nearly ready.

Due to the KeplerCam's good cosmetics, a bad-pixel mask was not required. The same $B V r^{\prime} i^{\prime}$ filters from CfA3 were used for CfA4. The second of the two CfA3 $U$ filters was used for CfA4 until it broke in 2009 January, and afterward an SDSS $u^{\prime}$ filter was used. A further description of the filters used in conjunction with the KeplerCam can be found at the FLWO Web site. ${ }^{15}$

\subsection{Observations}

The CfA Supernova Group depends on both professional and amateur SN searches for its observing targets. Most of these search surveys had typical limiting magnitudes of $19.5 \mathrm{mag}$. The $1.2 \mathrm{~m}$ telescope can reach targets north of declination $-20^{\circ}$. The CfA4 discovery data are displayed in Table 1 . The reported SN positions are a weighted mean from our subtracted images, usually in $r^{\prime}$, and are usually an improvement over the announced discovery positions. These positions will be of use for studies requiring more accurate SN positions, such as exploring the host-galaxy properties at the point of explosion. For the reader's convenience we also list the redshift, hostgalaxy name, and Milky Way color excess for each SN.

As explained in H09, the CfA Supernova Group rapidly acquires spectra of many of the new SNe brighter than $\sim 18.5 \mathrm{mag}$ and northward of $-20^{\circ}$ to provide typing and follow-up investigation. We also begin taking optical and $J H K$ photometry. This combination allows for a richer understanding of both individual SNe and the sample as a whole. Priority was usually given to younger and more interesting SNe.

\footnotetext{
14 http://linmax.sao.arizona.edu/FLWO/48/kepccd.html

15 http://linmax.sao.arizona.edu/FLWO/48/CCD.filters.html
}

The SNe in our sample come from a variety of SN searches. In many cases, the $\mathrm{SNe}$ are detected in galaxies that are targeted for monitoring. This means that the host galaxies do not constitute an unbiased sample of the universe, and the properties of the SN in this sample and of their hosts are not necessarily representative. See $\mathrm{H} 09$ for further details.

\subsection{Pipeline: Reduction Stage}

Images underwent bias subtraction and flat fielding. Domescreen flats were used for $B V r^{\prime} i^{\prime}$, while twilight flats were used for $U u^{\prime}$. The $i^{\prime}$-band fringes were slightly larger than in CfA3, so fringe corrections were applied. Cosmic rays were removed in the same way as in CfA3.

The UCAC3 catalog (Zacharias 2010) was used to produce a linear astrometric solution for the vast majority of the CfA4 images. The USNO-B1.0 (Monet 2003) or USNO-A2.0 catalogs (Monet 1998) were employed in the few cases where the UCAC3 catalog was too sparse. SWarp (Bertin et al. 2002) was run to properly scale and align the images. DoPHOT (Schechter et al. 1993) was then used to calculate fluxes for all stellar-shaped objects.

\subsection{Calibration}

We used Landolt (1992) to calibrate our $U B V$ bands and Smith et al. (2002) to calibrate our $r^{\prime} i^{\prime}$ bands. For our $u^{\prime}$ calibration we transformed the Landolt (1992) $U$ magnitudes into $u^{\prime}$ via the equation $u^{\prime}=U+0.854$ (Chonis \& Gaskell 2008).

As in CfA3, we performed aperture photometry on the Landolt/Smith standard stars and on our SN-field comparison stars using the NOAO/DIGIPHOT/APPHOT package in IRAF (Tody 1993). Comparison stars were chosen so that they were reasonably well isolated. Due to the deteriorated mirror, which resulted in larger stellar point-spread functions (PSFs), an aperture with radius of 18 pixels was used on both the standard and comparison stars. This was larger than the 15 pixels used in CfA3. An aperture correction was calculated from as many as four bright and isolated stars by subtracting the 6-pixelradius-aperture magnitude from the 18-pixel-radius-aperture magnitude and applied to the 6-pixel-radius magnitude of all of the stars in the field.

As in CfA3, a linear photometric transformation solution for each night was calculated from our Landolt/Smith stars using the system of Equations (1).

$$
\begin{aligned}
u-b & =\mathrm{zp}_{U B}+\alpha_{U B} x+\beta_{U B}(U-B) \\
b-v & =\mathrm{zp}_{B V}+\alpha_{B V} x+\beta_{B V}(B-V) \\
v-V & =\mathrm{zp}_{V}+\alpha_{V} x+\beta_{V}(B-V) \\
v-r & =\mathrm{zp}_{V r^{\prime}}+\alpha_{V r^{\prime}} x+\beta_{V r^{\prime}}\left(V-r^{\prime}\right) \\
v-i & =\mathrm{zp}_{V i^{\prime}}+\alpha_{V i^{\prime}} x+\beta_{V i^{\prime}}\left(V-i^{\prime}\right) .
\end{aligned}
$$

The terms on the left side of the equations are the instrumental colors except for the $V$-band term. The first term on the right side of each equation is the zero point, followed by the airmass coefficients, $\alpha$, times the airmass, $x$. The $V$-band equation is unique in that it directly relates the instrumental magnitude $v$ to the standard-system magnitude and color, $V$ and $B-V$. The other four equations only relate the instrumental and standardsystem colors to each other. The final term on the right of the four color equations multiplies the standard-system color of the standard stars by a coefficient, $\beta$, to convert the standard-system color into the natural-system color. When the $u^{\prime}$ filter replaced the $U$ filter, we used the above equations, replacing $U$ with $u^{\prime}$. 
Table 1

SN Ia Discovery Data

\begin{tabular}{|c|c|c|c|c|c|c|c|}
\hline SN Ia & Position & Galaxy & $z_{\text {helio }}$ & $z_{\mathrm{CMB}}$ & $E(B-V)$ & $d E(B-V)$ & Discovery Reference \\
\hline $2006 \mathrm{ct}$ & $12: 09: 56.851+47: 05: 44.31$ & 2MASX_J12095669+4705461 & 0.0315 & 0.0322 & 0.0191 & 0.0014 & IAUC 8720 \\
\hline 2006ou & $11: 37: 13.039+15: 26: 06.59$ & UGC_6588 & 0.0135 & 0.0146 & 0.0334 & 0.0016 & IAUC 8781 \\
\hline 2007A & $00: 25: 16.681+12: 53: 12.78$ & NGC_105 & 0.0177 & 0.0165 & 0.0736 & 0.0019 & CBET 795 \\
\hline 2007aj & $12: 47: 54.524+54: 00: 38.08$ & CGCG_270-24 & 0.0110 & 0.0115 & 0.0163 & 0.0016 & IAUC 8822 \\
\hline $2007 \mathrm{bj}$ & $16: 22: 10.589-01: 30: 51.33$ & NGC_6172 & 0.0167 & 0.0170 & 0.1177 & 0.0029 & IAUC 8834 \\
\hline $2007 \mathrm{cb}$ & $13: 58: 17.199-23: 22: 21.68$ & ESO_510-G31 & 0.0366 & 0.0375 & 0.0719 & 0.0011 & IAUC 8843 \\
\hline $2007 \mathrm{cc}$ & $14: 08: 42.050-21: 35: 47.50$ & ESO_578-G26 & 0.0291 & 0.0300 & 0.0794 & 0.0030 & IAUC 8843 \\
\hline $2007 \mathrm{cf}$ & $15: 23: 07.676+08: 31: 45.79$ & CGCG_77-100 & 0.0329 & 0.0335 & 0.0343 & 0.0007 & IAUC 8843 \\
\hline $2007 \mathrm{cn}$ & $22: 13: 55.790+13: 45: 23.45$ & UGC_11953 & 0.0253 & 0.0241 & 0.0621 & 0.0003 & IAUC 8851 \\
\hline $2007 \mathrm{cs}$ & $23: 49: 38.930+29: 55: 52.61$ & UGC_12798 & 0.0176 & 0.0164 & 0.0662 & 0.0022 & CBET 986 \\
\hline $2007 \mathrm{ev}$ & $22: 40: 06.201+24: 41: 56.67$ & AGC_320702 & 0.0427 & 0.0416 & 0.0490 & 0.0006 & CBET 991 \\
\hline $2007 \mathrm{fb}$ & $23: 56: 52.383+05: 30: 31.90$ & UGC_12859 & 0.0180 & 0.0168 & 0.0556 & 0.0011 & IAUC 8864 \\
\hline $2007 \mathrm{fq}$ & $20: 34: 55.742-23: 06: 15.38$ & MCG_-04-48-019 & 0.0425 & 0.0416 & 0.0420 & $\ldots$ & CBET 1001 \\
\hline $2007 \mathrm{fs}$ & $22: 01: 40.450-21: 30: 30.22$ & ESO_601-G5 & 0.0172 & 0.0162 & 0.0336 & 0.0007 & IAUC 8864 \\
\hline 2007hg & $04: 08: 32.676+02: 22: 43.20$ & [ISI96]_0405+0214 & 0.0300 & 0.0297 & 0.3799 & 0.0106 & CBET 1047 \\
\hline $2007 \mathrm{hj}$ & $23: 01: 47.880+15: 35: 11.23$ & NGC_7461 & 0.0141 & 0.0129 & 0.0883 & 0.0120 & IAUC 8874 \\
\hline 2007hu & $16: 56: 29.887+27: 58: 39.75$ & NGC_6261 & 0.0354 & 0.0354 & 0.0458 & 0.0023 & CBET 1056 \\
\hline 2007if & $01: 10: 51.370+15: 27: 39.63$ & [YQ2007]_J011051.37+152739.9 & 0.0742 & 0.0731 & 0.0831 & 0.0066 & CBET 1059 \\
\hline 2007ir & $02: 33: 41.898+37: 40: 08.12$ & UGC_2033 & 0.0352 & 0.0345 & 0.0495 & 0.0009 & CBET 1067 \\
\hline 2007 is & $16: 47: 14.607+40: 14: 36.40$ & UGC_10553 & 0.0297 & 0.0297 & 0.0201 & 0.0012 & IAUC 8874 \\
\hline 2007jg & $03: 29: 50.815+00: 03: 24.55$ & SDSS_J032950.83+000316.0 & 0.0371 & 0.0366 & 0.1065 & 0.0023 & CBET 1076 \\
\hline $2007 \mathrm{kd}$ & $09: 25: 58.041+34: 38: 00.11$ & MCG_+06-21-36 & 0.0242 & 0.0250 & 0.0217 & 0.0005 & IAUC 8874 \\
\hline $2007 \mathrm{kf}$ & $17: 31: 31.266+69: 18: 39.59$ & [K2007]J173131.76+691840.1 & 0.0460 & 0.0458 & 0.0439 & 0.0011 & IAUC 8875 \\
\hline $2007 \mathrm{~kg}$ & $23: 58: 37.493+60: 59: 07.41$ & 2MFGC_18005 & 0.0070 & 0.0063 & 0.9977 & 0.0238 & IAUC 8875 \\
\hline $2007 \mathrm{kh}$ & $03: 15: 12.049+43: 10: 13.39$ & [YAA2007a]J031512.10+431013.0 & 0.0500 & 0.0495 & 0.1984 & 0.0030 & CBET 1089 \\
\hline $2007 \mathrm{kk}$ & $03: 42: 23.258+39: 14: 30.30$ & UGC_2828 & 0.0410 & 0.0406 & 0.2291 & 0.0132 & CBET 1096 \\
\hline 2007le & $23: 38: 48.452-06: 31: 21.83$ & NGC_7721 & 0.0067 & 0.0055 & 0.0334 & 0.0003 & CBET 1100 \\
\hline 2007nq & $00: 57: 33.721-01: 23: 20.29$ & UGC_595 & 0.0450 & 0.0439 & 0.0354 & 0.0012 & CBET 1106 \\
\hline $2007 \mathrm{ob}$ & $23: 12: 25.988+13: 54: 49.13$ & 2MASX_J23122598+1354503 & 0.0339 & 0.0327 & 0.0681 & 0.0015 & CBET 1112 \\
\hline $2007 r x$ & $23: 40: 11.782+27: 25: 15.59$ & BATC_J234012.05+272512.23 & 0.0301 & 0.0289 & 0.0890 & 0.0078 & CBET 1157 \\
\hline 2007 ss & $12: 41: 06.150+50: 23: 28.51$ & NGC_4617 & 0.0155 & 0.0161 & 0.0149 & 0.0004 & CBET 1175 \\
\hline $2007 \mathrm{su}$ & $22: 19: 08.884+13: 10: 39.89$ & SDSS_J221908.85+131040.5 & 0.0279 & 0.0267 & 0.0830 & 0.0006 & CBET 1178 \\
\hline 2007 sw & $12: 13: 36.933+46: 29: 36.56$ & UGC_7228 & 0.0252 & 0.0260 & 0.0186 & 0.0014 & CBET 1185 \\
\hline 2007ux & 10:09:19.939 + 14:59:33.07 & 2MASX_J10091969+1459268 & 0.0309 & 0.0320 & 0.0448 & 0.0008 & CBET 1187 \\
\hline 2008A & $01: 38: 17.394+35: 22: 13.06$ & NGC_634 & 0.0165 & 0.0156 & 0.0542 & 0.0024 & CBET 1193 \\
\hline 2008C & $06: 57: 11.469+20: 26: 13.58$ & UGC_3611 & 0.0166 & 0.0171 & 0.0839 & 0.0026 & CBET 1195 \\
\hline 2008Q & $01: 24: 57.207+09: 33: 01.30$ & NGC_524 & 0.0600 & 0.0590 & 0.0828 & 0.0010 & CBET 1228 \\
\hline $2008 Y$ & $11: 19: 30.581+54: 27: 46.21$ & MCG_+9-19-39 & 0.0697 & 0.0703 & 0.0129 & 0.0009 & CBET 1240 \\
\hline $2008 Z$ & $09: 43: 15.258+36: 17: 03.64$ & SDSS_J094315.36+361709.2 & 0.0210 & 0.0218 & 0.0114 & 0.0007 & CBET 1243 \\
\hline $2008 \mathrm{ac}$ & $11: 53: 45.200+48: 25: 20.79$ & SDSS_J115345.22+482521.0 & 0.0528 & 0.0535 & 0.0190 & 0.0003 & CBET 1245 \\
\hline 2008ad & $12: 49: 37.071+28: 19: 45.82$ & ROTSE_J124936.88+281944.8 & 0.0500 & 0.0509 & 0.0130 & 0.0010 & CBET 1245 \\
\hline $2008 \mathrm{ae}$ & $09: 56: 03.160+10: 29: 58.52$ & IC_577 & 0.0301 & 0.0312 & 0.0277 & 0.0007 & CBET 1247 \\
\hline 2008ai & $10: 57: 39.957+37: 39: 41.40$ & CGCG_184-39 & 0.0353 & 0.0361 & 0.0163 & 0.0010 & CBET 1256 \\
\hline 2008ar & $12: 24: 37.922+10: 50: 16.74$ & IC_3284 & 0.0261 & 0.0272 & 0.0373 & 0.0013 & CBET 1273 \\
\hline 2008at & $10: 27: 12.469+71: 24: 55.55$ & UGC05645 & 0.0350 & 0.0352 & 0.0912 & 0.0025 & CBET 1277 \\
\hline 2008bi & $08: 35: 53.388+00: 42: 22.85$ & NGC_2618 & 0.0134 & 0.0144 & 0.0441 & 0.0014 & CBET 1312 \\
\hline 2008bw & $18: 26: 50.440+51: 08: 16.42$ & UGC_11241 & 0.0331 & 0.0328 & 0.0399 & 0.0017 & CBET 1346 \\
\hline 2008by & $12: 05: 20.907+40: 56: 44.43$ & SDSS_J120520.81+405644.4 & 0.0450 & 0.0458 & 0.0135 & 0.0002 & CBET 1350 \\
\hline $2008 \mathrm{bz}$ & $12: 38: 57.686+11: 07: 45.60$ & 2MASX_J12385810+1107502 & 0.0603 & 0.0614 & 0.0269 & 0.0021 & CBET 1353 \\
\hline $2008 \mathrm{~cd}$ & $13: 15: 01.777-15: 57: 06.70$ & NGC_5038 & 0.0074 & 0.0085 & 0.0688 & 0.0002 & CBET 1360 \\
\hline $2008 \mathrm{cf}$ & $14: 07: 32.585-26: 33: 07.74$ & [WLF2008]_J140732.38-263305.6 & 0.0460 & 0.0469 & 0.0674 & 0.0017 & CBET 1365 \\
\hline $2008 \mathrm{~cm}$ & $13: 29: 12.826+11: 16: 20.65$ & NGC_2369 & 0.0111 & 0.0116 & 0.1139 & 0.0013 & CBET 1384 \\
\hline $2008 \mathrm{dr}$ & $22: 10: 51.664+02: 06: 29.34$ & NGC_7222 & 0.0414 & 0.0403 & 0.0428 & 0.0009 & CBET 1419 \\
\hline $2008 d s$ & $00: 29: 50.820+31: 23: 33.88$ & UGC_299 & 0.0210 & 0.0200 & 0.0643 & 0.0028 & CBET 1419 \\
\hline $2008 \mathrm{dt}$ & $16: 56: 30.592+27: 58: 33.83$ & NGC_6261 & 0.0354 & 0.0354 & 0.0458 & 0.0021 & CBET 1423 \\
\hline $2008 \mathrm{fr}$ & $01: 11: 49.224+14: 38: 26.21$ & SDSS_J011149.19+143826.5 & 0.0490 & 0.0479 & 0.0449 & 0.0014 & CBET 1513 \\
\hline $2008 \mathrm{gb}$ & $02: 57: 57.141+46: 51: 56.19$ & UGC_2427 & 0.0370 & 0.0364 & 0.1983 & 0.0041 & CBET 1527 \\
\hline $2008 \mathrm{gl}$ & $01: 20: 54.820+04: 48: 19.22$ & UGC_881 & 0.0340 & 0.0330 & 0.0284 & 0.0009 & CBET 1545 \\
\hline 2008hj & 00:04:01.913-11:10:08.35 & MCG_-2-1-14 & 0.0379 & 0.0367 & 0.0361 & 0.0009 & CBET 1579 \\
\hline 2008hm & $03: 27: 10.889+46: 56: 39.20$ & 2MFGC_2845 & 0.0197 & 0.0192 & 0.4425 & 0.0099 & CBET 1586 \\
\hline $2008 \mathrm{hs}$ & $02: 25: 29.594+41: 50: 34.92$ & NGC_910 & 0.0173 & 0.0166 & 0.0573 & 0.0004 & CBET 1598 \\
\hline 2008hv & $09: 07: 34.066+03: 23: 32.18$ & NGC_2765 & 0.0125 & 0.0136 & 0.0321 & 0.0009 & CBET 1601 \\
\hline 2009D & $03: 54: 22.817-19: 10: 54.56$ & MCG_-03-10-52 & 0.0250 & 0.0247 & 0.0529 & 0.0014 & CBET 1647 \\
\hline 2009Y & $14: 42: 24.563-17: 14: 46.70$ & NGC_5728 & 0.0093 & 0.0101 & 0.1016 & 0.0006 & CBET 1684 \\
\hline 2009ad & $05: 03: 33.393+06: 39: 35.82$ & UGC_3236 & 0.0284 & 0.0283 & 0.1120 & 0.0013 & CBET 1694 \\
\hline 2009al & $10: 51: 22.049+08: 34: 41.98$ & NGC_3425 & 0.0221 & 0.0233 & 0.0246 & 0.0005 & CBET 1705 \\
\hline
\end{tabular}


Table 1

(Continued)

\begin{tabular}{|c|c|c|c|c|c|c|c|}
\hline SN Ia & Position & Galaxy & $z_{\text {helio }}$ & $z_{\mathrm{CMB}}$ & $E(B-V)$ & $d E(B-V)$ & Discovery Reference \\
\hline 2009an & $12: 22: 47.385+65: 51: 04.60$ & NGC_4332 & 0.0092 & 0.0095 & 0.0186 & 0.0003 & CBET 1707 \\
\hline $2009 b v$ & $13: 07: 20.517+35: 47: 03.20$ & MCG_+6-29-39 & 0.0367 & 0.0375 & 0.0086 & 0.0009 & CBET 1741 \\
\hline $2009 \mathrm{dc}$ & $15: 51: 12.083+25: 42: 28.43$ & UGC_10064 & 0.0214 & 0.0217 & 0.0696 & 0.0017 & CBET 1762 \\
\hline 2009do & $12: 34: 58.316+50: 51: 03.81$ & NGC_4537 & 0.0397 & 0.0403 & 0.0149 & 0.0006 & CBET 1778 \\
\hline $2009 \mathrm{ds}$ & $11: 49: 04.025-09: 43: 44.48$ & NGC_3905 & 0.0192 & 0.0204 & 0.0389 & 0.0007 & CBET 1784 \\
\hline $2009 \mathrm{fv}$ & $16: 29: 44.191+40: 48: 41.44$ & NGC_6173 & 0.0293 & 0.0294 & 0.0063 & 0.0014 & CBET 1834 \\
\hline $2009 \mathrm{gf}$ & $14: 15: 37.127+14: 16: 48.74$ & NGC_5525 & 0.0185 & 0.0193 & 0.0255 & 0.0007 & CBET 1844 \\
\hline 2009hp & $02: 58: 23.938+06: 35: 34.64$ & MCG_+01-08-30 & 0.0211 & 0.0204 & 0.2300 & $\ldots$ & CBET 1888 \\
\hline 2009ig & $02: 38: 11.613-01: 18: 45.52$ & NGC_1015 & 0.0088 & 0.0080 & 0.0320 & 0.0009 & CBET 1918 \\
\hline $2009 \mathrm{jr}$ & $20: 26: 26.013+02: 54: 31.73$ & IC_1320 & 0.0165 & 0.0156 & 0.1347 & 0.0035 & CBET 1964 \\
\hline $2009 \mathrm{kk}$ & $03: 49: 44.320-03: 15: 52.66$ & 2MFGC_3182 & 0.0129 & 0.0124 & 0.1376 & 0.0029 & CBET 1991 \\
\hline $2009 \mathrm{kq}$ & $08: 36: 15.148+28: 04: 01.67$ & $\mathrm{MCG}_{-}+5-21-1$ & 0.0116 & 0.0124 & 0.0410 & 0.0006 & CBET 2005 \\
\hline 2009le & 02:09:17.160-23:24:44.74 & ESO_478-6 & 0.0178 & 0.0170 & 0.0164 & 0.0006 & CBET 2022 \\
\hline $20091 f$ & $02: 01: 39.616+15: 19: 58.13$ & 2MASX_J02014081+1519521 & 0.0450 & 0.0441 & 0.0525 & 0.0023 & CBET 2023 \\
\hline 2009li & $00: 22: 51.395+06: 58: 11.35$ & IC_1549 & 0.0404 & 0.0392 & 0.0267 & 0.0010 & CBET 2026 \\
\hline 2009na & $10: 47: 01.444+26: 32: 37.73$ & UGC_5884 & 0.0210 & 0.0220 & 0.0319 & 0.0018 & CBET 2098 \\
\hline 2009nq & $23: 15: 17.004+19: 01: 21.58$ & NGC_7549 & 0.0158 & 0.0146 & 0.1455 & 0.0046 & CBET 2110 \\
\hline $2010 \mathrm{~A}$ & $02: 32: 39.459+00: 37: 09.90$ & UGC_2019 & 0.0207 & 0.0199 & 0.0291 & 0.0011 & CBET 2109 \\
\hline $2010 \mathrm{H}$ & $08: 06: 24.342+01: 02: 09.01$ & IC_494 & 0.0152 & 0.0160 & 0.0308 & 0.0011 & CBET 2130 \\
\hline $2010 Y$ & $10: 51: 03.994+65: 46: 46.40$ & NGC_3392 & 0.0109 & 0.0113 & 0.0135 & 0.0015 & CBET 2168 \\
\hline 2010ag & $17: 03: 53.653+31: 30: 06.70$ & UGC_10679 & 0.0338 & 0.0338 & 0.0309 & 0.0013 & CBET 2195 \\
\hline 2010ai & $12: 59: 24.005+27: 59: 47.13$ & SDSS_J125925.04+275948.2 & 0.0184 & 0.0193 & 0.0094 & 0.0012 & CBET 2200 \\
\hline $2010 \mathrm{cr}$ & $13: 29: 25.082+11: 47: 46.49$ & NGC_5177 & 0.0216 & 0.0225 & 0.0345 & 0.0014 & CBET 2281 \\
\hline 2010dt & $16: 43: 15.063+32: 40: 27.56$ & CGCG_168-029 & 0.0529 & 0.0529 & 0.0341 & 0.0011 & CBET 2307 \\
\hline $2010 \mathrm{dw}$ & $15: 22: 40.279-05: 55: 16.46$ & 2MASX_J15224062-0555214 & 0.0381 & 0.0387 & 0.0933 & 0.0011 & CBET 2310 \\
\hline SNF20080522-000 & $13: 36: 47.592+05: 08: 30.41$ & SDSS_J133647.59+050833.0 & 0.0472 & 0.0482 & 0.0265 & 0.0005 & SNF site \\
\hline SNF20080522-011 & $15: 19: 58.920+04: 54: 16.73$ & SDSS_J151959.16+045411.2 & 0.0397 & 0.0403 & 0.0427 & 0.0008 & SNF site \\
\hline PTF10bjs & $13: 01: 11.215+53: 48: 57.49$ & $\mathrm{MCG}_{-}+9-21-83$ & 0.0300 & 0.0306 & 0.0176 & 0.003 & ATEL 2453 \\
\hline
\end{tabular}

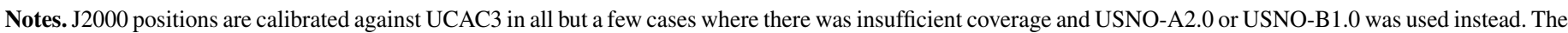

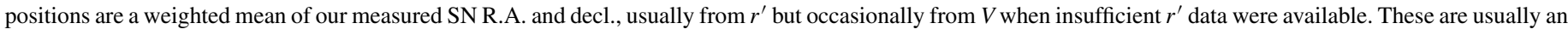

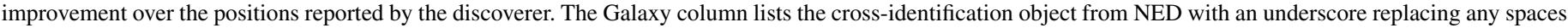

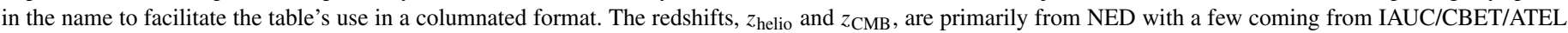

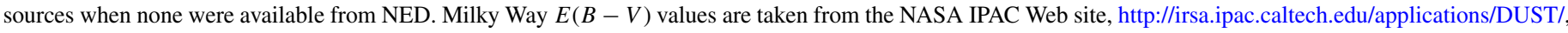

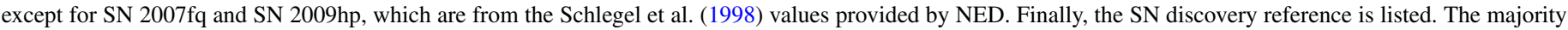
are from CBET, IAUC, or ATEL, while two SNe come from the SNF Web site, http://snfactory.lbl.gov/snf/open_access/snlist.php.

The photometric solution was applied to the comparison star measurements. This produced tertiary standards that were used to calibrate the SN measurements in the natural system. To calculate the photometric zero point for each SN image, we took a weighted mean of the differences between our calibrated magnitudes and the instrumental DoPHOT measurements of the comparison stars.

Most of our SN fields were observed on multiple photometric nights to ensure more accurate calibration. However, this was not always possible. All SN fields that were calibrated on only one night had other SN fields calibrated on the same night that were consistent across multiple nights. This increases confidence but does not guarantee the single-night calibrations. The comparison star uncertainties include the measurement uncertainties, the standard deviation of measurements from multiple nights (for single nights, an appropriate error floor was used instead), and the uncertainty of the transformation to the standard system. The typical uncertainty of our $V$-band comparison star measurements is $0.015 \mathrm{mag}$.

The color coefficients from each photometric night are plotted in Figure 1 . The $V, V-i^{\prime}, U-B$, and $u^{\prime}-B$ coefficients do not show any significant trend over time and can be fit well by one average value, while the $B-V$ and $V-r^{\prime}$ coefficients show a step-function distribution with one value in the period before mid-2009 (period one) and another value in the period after
Table 2

Photometric Color Terms

\begin{tabular}{lccc}
\hline \hline Filter/Time Period & Color Term & Value & Nights \\
\hline$U-B /$ both periods & $(u-b) /(U-B)$ & $0.9981 \pm 0.0209$ & 17 \\
$u^{\prime}-B /$ both periods & $(u-b) /\left(u^{\prime}-B\right)$ & $0.9089 \pm 0.0057$ & 28 \\
$B-V /$ period one & $(b-v) /(B-V)$ & $0.9294 \pm 0.0026$ & 38 \\
$B-V /$ period two & $(b-v) /(B-V)$ & $0.8734 \pm 0.0024$ & 25 \\
$V /$ both periods & $(v-V) /(B-V)$ & $0.0233 \pm 0.0018$ & 63 \\
$V-r^{\prime} /$ period one & $(v-r) /\left(V-r^{\prime}\right)$ & $1.0684 \pm 0.0028$ & 38 \\
$V-r^{\prime} /$ period two & $(v-r) /\left(V-r^{\prime}\right)$ & $1.0265 \pm 0.0033$ & 25 \\
$V-i^{\prime} /$ both periods & $(v-i) /\left(V-i^{\prime}\right)$ & $1.0239 \pm 0.0016$ & 63 \\
\end{tabular}

Notes. Lowercase $u b v r i$ refer to the instrumental magnitudes, while $U u^{\prime} B V r^{\prime} i^{\prime}$ refer to the standard magnitudes. All color terms implicitly contain an additive constant. For example, $(v-V)=0.0233(B-V)+$ const, $(v-i)=$ $1.0239\left(V-i^{\prime}\right)+$ const.

(period two). We chose to use 2009 August $15(\mathrm{MJD}=55058)$ as the dividing point and calculated average $B-V$ and $V-r^{\prime}$ color coefficients for period one and another average for period two. The color coefficients are listed in Table 2 . The largest difference between the two periods was in the $B-V$ color coefficients, which decreased from 0.93 to 0.87 . Since the $V$-band color 


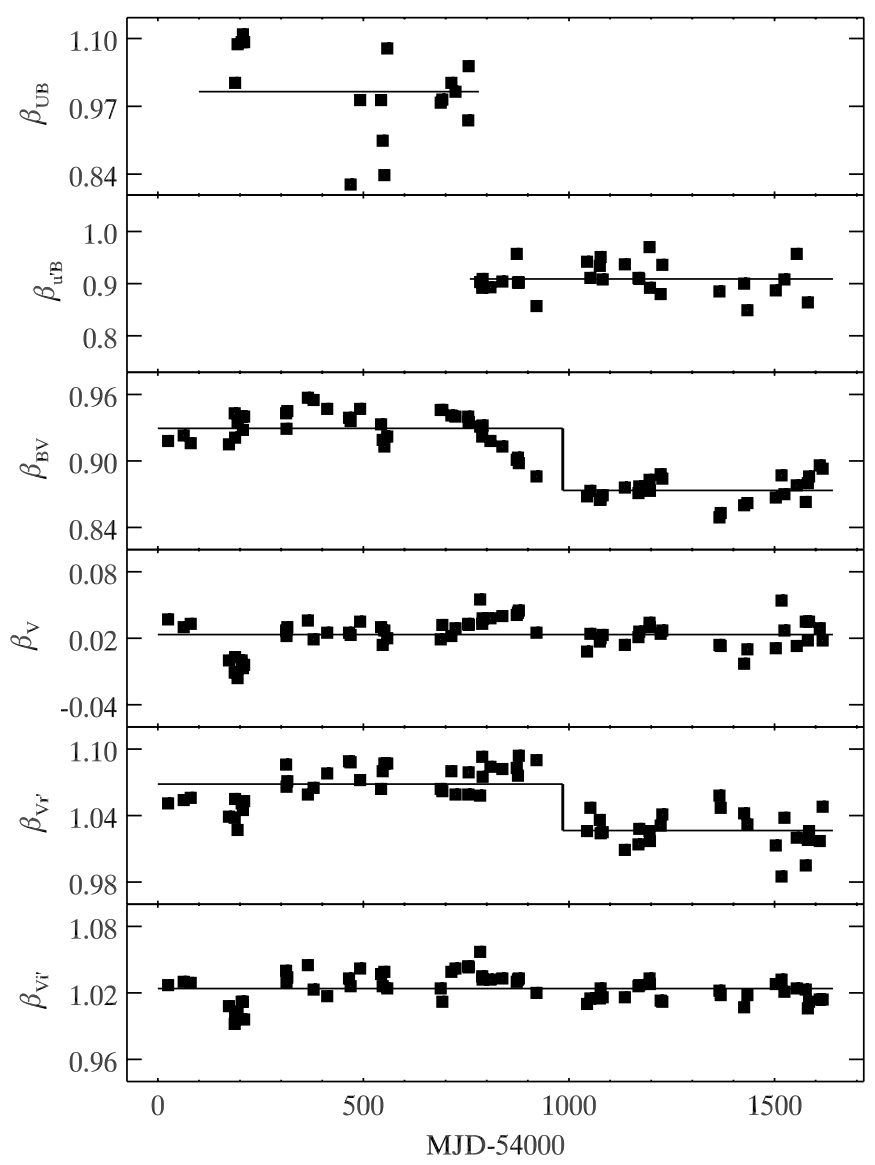

Figure 1. $U u^{\prime} B V r^{\prime} i^{\prime}$ color coefficients are plotted vs. time with the average value over the relevant time periods shown as a solid line. The $v-V$ and $V-i^{\prime}$ coefficients are sufficiently stable to be represented by one constant value across the whole time domain, while the $B-V$ and $V-r^{\prime}$ coefficients are each better described by one value in period one and another value in period two. The $U-B$ coefficients have large uncertainties (not shown) and a large scatter, while the $u^{\prime}-B$ coefficients have a much smaller scatter, illustrating the superior precision of $u^{\prime}$ measurements.

coefficient was stable across the two time periods, this implies that the $B$ passband shifted redward in period two. Deposits or condensation on the camera are likely causes for the changing color coefficients. The KeplerCam was baked on 2011 May 17 to remove deposits/condensation, and the $B-V$ and $V-r^{\prime}$ color coefficients derived after this returned to their period-one values. However, these post-baking photometric nights were not used for any CfA4 photometric calibration, so only the two time periods, one before and one after 2009 August, with their respective color coefficients were needed. In addition to this, the $1.2 \mathrm{~m}$ mirror was deteriorating from 2007 to 2011 , losing about 0.6 mag of sensitivity in $V$. We note that the KeplerCam underwent regular bakeouts every August, but none of these produced a dramatic difference like the one in 2011 May. There is no evidence that the 2011 bakeout procedure was different from previous cycles of desiccation and cleaning, but the result was a significant change in the color coefficients. Whatever the cause, the $B-V$ and $V-r^{\prime}$ color coefficients returned to their period-one values, and the most likely explanation is that the 2011 May bakeout removed deposits/condensation that previous ones could not.

Synthesized natural-system $B V r^{\prime} i^{\prime}$ passbands for the KeplerCam were calculated in $\mathrm{H} 09$ by combining the primary and secondary mirror reflectivities (taken as the square of the measured

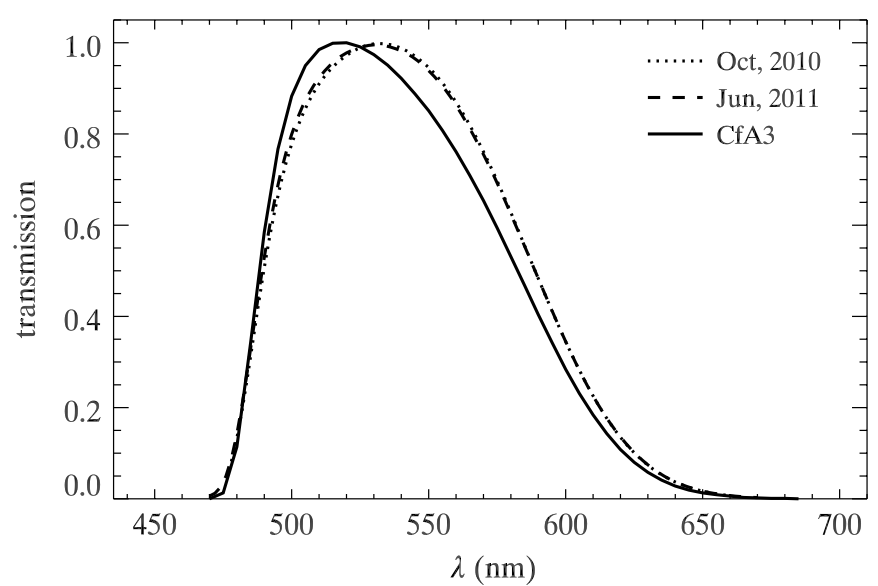

Figure 2. C12 $V$ passbands measured before (dotted) and after (dashed) the 2011 May bakeout are highly consistent with each other and in reasonable agreement with the CfA3 synthetic $V$ passband (solid).

reflectivity of the primary), the measured filter transmissions, and the measured KeplerCam quantum efficiencies. No atmospheric component was included. These passbands were presented as normalized photon sensitivities. No $U$-band passband was made due to a lack of a $U$ filter transmission curve.

More recently, C. Cramer et al. (2012, in preparation, hereafter C12) measured the FLWO $1.2 \mathrm{~m}$ KeplerCam $B V r^{\prime} i^{\prime}$ passbands using the technique initially described in Woodward et al. (2010) and Stubbs \& Tonry (2006). The dome screen was illuminated with light from a monochromatic, tunable source to generate a series of monochromatic dome flats spanning each filter passband. A NIST-calibrated photodiode ${ }^{16}$ monitored the total amount of light incident on the telescope during each exposure. Filter passbands were generated by scaling the camera response to the photodiode signal. The measured passbands therefore include not only the filters, camera, and mirrors, but also the effect of other optics in the telescope optical train-notably, a doublet corrector lens with an aging anti-reflective coating on each of the four surfaces - as well as accumulated dirt and condensation in the camera. The $V r^{\prime} i^{\prime}$ passbands were measured four times: in 2010 July and October and 2011 April and June. The $B$ passband was measured in 2010 October and 2011 April and June. We therefore have measured passbands both before and after the 2011 May bakeout. For more details and tables of the measured passbands, see $\mathrm{C} 12$.

The C12 $V r^{\prime} i^{\prime}$ passbands are relatively stable across the bakeout and agree reasonably well with the synthesized CfA3 $V r^{\prime} i^{\prime}$ passbands (see Figure 2 for $V$ ), but the C12 pre-bakeout $B$ passband is significantly redward of both its post-bakeout counterpart and the synthesized CfA3 $B$ passband, as seen in Figure 3 . The pre-bakeout $\mathrm{C} 12$ passbands were observed at two separate times, six months apart, and were virtually identical. The period-two color coefficients were also stable over this same range of time (and longer). The implication is that the pre-bakeout $\mathrm{C} 12$ passbands are valid over the period-two time range where the deposits/condensation were present.

After the bakeout, the $\mathrm{C} 12 \mathrm{~B}$ passband shifted blueward while the $\mathrm{C} 12 \mathrm{~V}$ passband remained stable. This is consistent with the increase of the $B-V$ color coefficients back to the period-one 0.93 level. The post-bakeout $\mathrm{C} 12 \mathrm{~B}$ passband can also be seen to be much more consistent with the synthesized CfA $3 B$ passband. We also point out that the CfA3 $B-V$ color coefficient was

\footnotetext{
$\overline{16}$ http://www.nist.gov/calibrations/upload/sp250-41a.pdf
} 


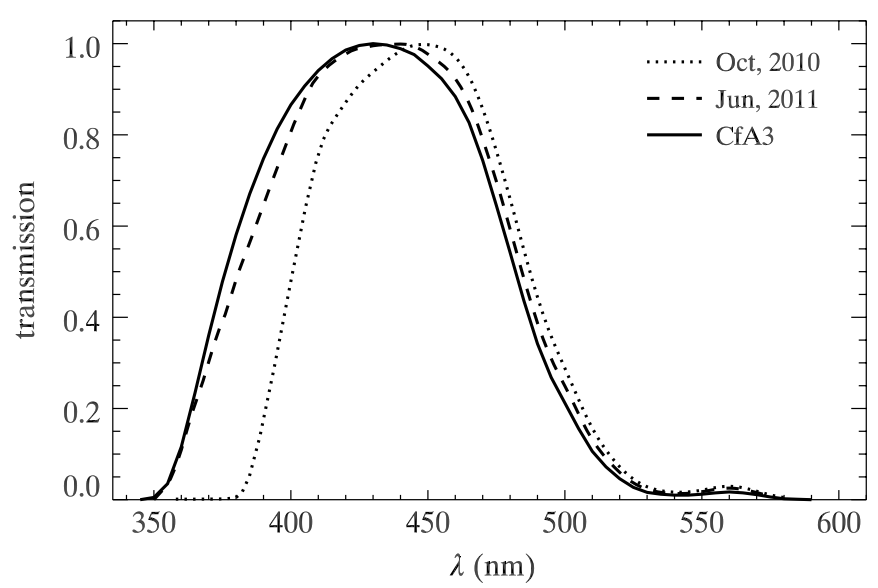

Figure 3. C12 B passbands measured before (dotted) and after (dashed) the 2011 May bakeout, showing the blueward shift caused by the bakeout. The CfA3 synthetic $B$ passband (solid) agrees fairly well with the post-bakeout $\mathrm{C} 12$ $B$ passband.

0.92, fairly close to the CfA4 period-one value. These pieces of information suggest that the post-bakeout $\mathrm{C} 12 \mathrm{~B}$ passband can be used as the CfA4 period-one natural-system $B$ passband.

To summarize, the $\mathrm{C} 12$ post-bakeout passbands should be used in conjunction with the CfA4 period-one natural-system light curves, while the $\mathrm{C} 12$ pre-bakeout passbands should be used for period two. Also, due to the reasonable consistency of the $B V r^{\prime} i^{\prime} \mathrm{CfA} 3$ and CfA4 period-one color coefficients, the $\mathrm{C} 12$ post-bakeout passbands can be used with the CfA3 natural-system light curves. The similarity of the synthesized CfA3 passbands to the $\mathrm{C} 12 V r^{\prime} i^{\prime}$ and post-bakeout $B$ passbands suggests that they were sufficiently accurate and that their use in cosmological studies was satisfactory (Amanullah et al. 2010; Suzuki et al. 2012; Sullivan et al. 2011).

\subsection{Pipeline: Host-galaxy Subtraction}

Each of the $94 \mathrm{SNe}$ in the CfA4 sample underwent hostgalaxy subtraction. Reference images were acquired on clear nights with good seeing and little or no moon so as to maximize their signal-to-noise ratio. However, due to the poor mirror quality, the images had larger PSF sizes than in CfA3. To combat this, multiple reference images were subtracted from the majority of the data images.

The same subtraction algorithm and software as in CfA3 were used for CfA4. A convolution kernel that transforms the PSF of one image to the PSF of the other was calculated using the algorithm of Alard \& Lupton (1998) and Alard (2000), with slight improvements as in Becker et al. (2004) and Miknaitis et al. (2007), and then the subtraction was performed. The SN flux in the difference image was then measured with the DoPHOT PSF from the stars of the unconvolved image.

The natural-system flux normalization for the difference image was chosen from the $\mathrm{SN}$ data image as opposed to the reference image. This ensured that the normalization of the SN data would be consistent with the passbands and color coefficients from the same time period.

Noise maps were propagated for both data and reference images and used to calculate a noise map for the difference image. Information from the noise image was combined with the DoPHOT uncertainty and calibration uncertainty to produce the uncertainty of the natural-system SN measurement.

The subtraction process was not always perfect, and this introduced extra uncertainty. Steps were taken to estimate this uncertainty. In the cases where multiple reference images existed and were successfully subtracted from one data image, we had a distribution of values that provided a better estimate of the true SN flux and its uncertainty. The differences in these values were due to differences in the various reference images. In order to arrive at one final light-curve point, the multiple photometry values from each data image were plotted and any extreme outliers were removed. Suppose that $N$ values remain. They are different from each other due to Poisson noise in the host galaxy and sky flux in the reference images and due to slight limitations in convolving every reference image equally well to the data image. We took the median of these $N$ values to be the final light-curve data point. There are also $N$ photometrypipeline uncertainties associated with each of the $N$ photometry values. To calculate the final light-curve uncertainty for the data point in question, $\sigma_{\text {total }}$, the median of the $N$ photometrypipeline uncertainties (we will call this median $\sigma_{\text {pipe }}$ ) was added in quadrature to the standard deviation of the $N$ photometry values $\left(\sigma_{\text {phot }}\right)$.

Formally, there is a slight double counting of the Poisson noise of the reference images since it is part of the standard deviation of the $N$ photometry values, $\sigma_{\text {phot }}$, and is also included in the difference image noise maps. However, this is dwarfed by the size of the other uncertainties and has no significant effect on the size of the final error bars. In $\sigma_{\text {phot }}$ the Poisson noise of the reference image is typically much smaller than the uncertainties due to imperfect subtractions. And in the pipeline uncertainty, the combination of the data image noise with the DoPhot and calibration uncertainties is larger than the reference image noise that is taken during dark time with better seeing.

For the cases where only one reference image was successfully subtracted, the light-curve value was simply the singlesubtraction value. The uncertainty from the single-subtraction photometry was added in quadrature to an estimate of what the standard deviation would have been had multiple reference images existed. This estimate was based on a quadratic fit of the standard deviations of the multiple-subtraction photometry values versus $\mathrm{SN}$ magnitude in a given band at magnitudes fainter than 16 mag. For magnitudes brighter than this a constant but representative value was used. This derived function then gave a reasonable estimate that was easily calculated from the SN magnitude of the single-subtraction point. This function is presented in Table 3.

Having multiple reference images for most of the CfA4 sample is one of the main differences with CfA3. In CfA3, there was only one reference image for most of the SNe and none for some SNe with faraway hosts. The uncertainties for the single reference-image CfA3 photometry were what are here called $\sigma_{\text {pipe }}$ for $N=1$ and are almost certainly an underestimate of the true uncertainty. Future use of the CfA3 sample would benefit by adding an estimate of $\sigma_{\text {phot }}$. The function we derived for the CfA4 single reference-image photometry could serve as such an estimate and can be added in quadrature to the quoted CfA3 uncertainties.

It was found that the standard deviation of the $N$ light-curve values was typically on the order of their photometry-pipeline uncertainties. Using multiple reference images increased the accuracy of both the CfA4 light-curve values and their uncertainties. We believe that the final error bars are our best estimate of the true uncertainties.

Our light curves were produced in the natural system and then converted to the standard system by using the color coefficients in Table 2. Figures 4-7 show four of the better-sampled CfA4 
Table 3

Estimation of $\sigma_{\text {phot }}$ for Single Reference-Image Photometry

\begin{tabular}{lcccccc}
\hline \hline Mag & $U$ & $u^{\prime}$ & $B$ & $V$ & $r^{\prime}$ & $i^{\prime}$ \\
\hline$\leqslant 16.0$ & 0.020 & 0.020 & 0.012 & 0.010 & 0.010 & 0.010 \\
16.5 & 0.030 & 0.038 & 0.016 & 0.013 & 0.012 & 0.013 \\
17.0 & 0.048 & 0.058 & 0.024 & 0.019 & 0.018 & 0.019 \\
17.5 & 0.073 & 0.081 & 0.037 & 0.028 & 0.026 & 0.028 \\
18.0 & 0.105 & 0.107 & 0.053 & 0.041 & 0.037 & 0.041 \\
18.5 & 0.144 & 0.136 & 0.074 & 0.058 & 0.052 & 0.056 \\
19.0 & 0.191 & 0.168 & 0.099 & 0.077 & 0.070 & 0.075 \\
19.5 & 0.245 & 0.203 & 0.128 & 0.100 & 0.091 & 0.097 \\
20.0 & 0.306 & 0.240 & 0.162 & 0.126 & 0.115 & 0.122 \\
20.5 & 0.374 & 0.281 & 0.199 & 0.156 & 0.142 & 0.150 \\
21.0 & 0.450 & 0.324 & 0.241 & 0.189 & 0.172 & 0.181 \\
\hline
\end{tabular}

Notes. This estimate of $\sigma_{\text {phot }}$ for the $N=1$ cases was based on a quadratic fit of the standard deviations of the multiple-subtraction photometry values versus SN magnitude in a given band at magnitudes fainter than 16 mag. For magnitudes brighter than this a constant but representative value was used. The CfA 3 photometry uncertainties would be improved by adding these values in quadrature.

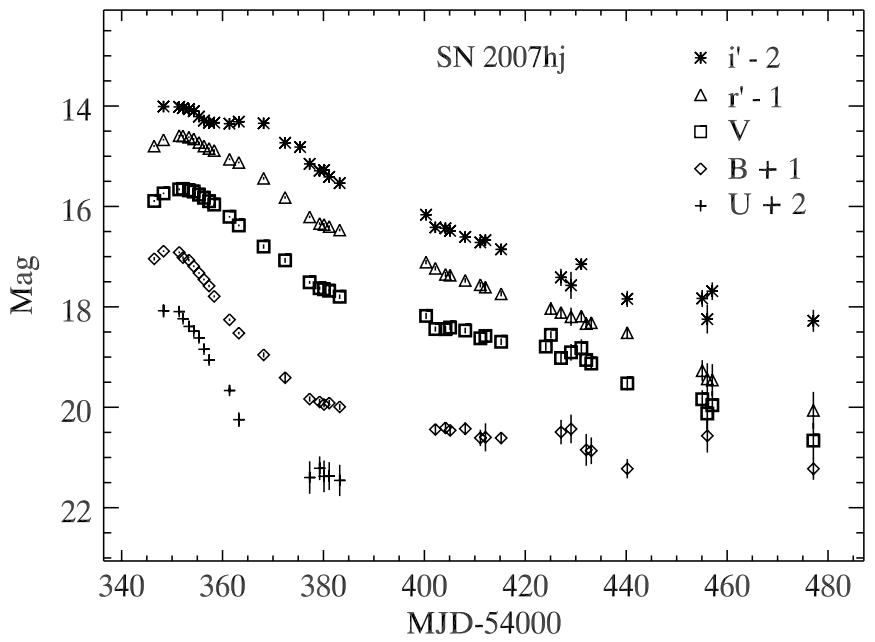

Figure 4. SN 2007hj, one of the better-sampled CfA4 light curves. The error bars are smaller than the symbols for most of the data points.

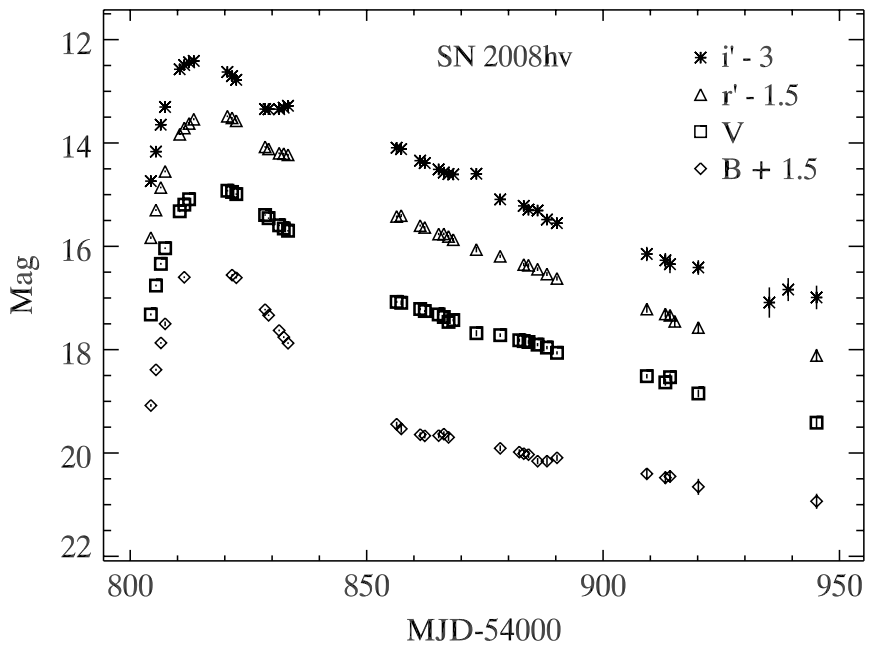

Figure 5. SN 2008hv, one of the better-sampled CfA4 light curves. The error bars are smaller than the symbols for most of the data points.

light curves. The standard-system comparison stars and both natural- and standard-system light curves for all the objects can be found in the online version of Tables 4-6 and at our

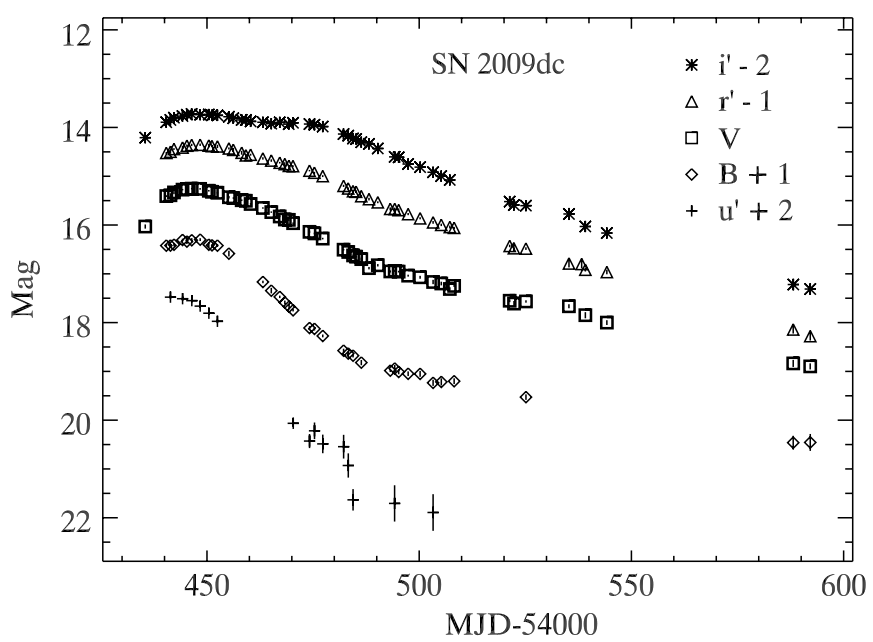

Figure 6. SN 2009dc, one of the better-sampled CfA4 light curves. The error bars are smaller than the symbols for most of the data points.

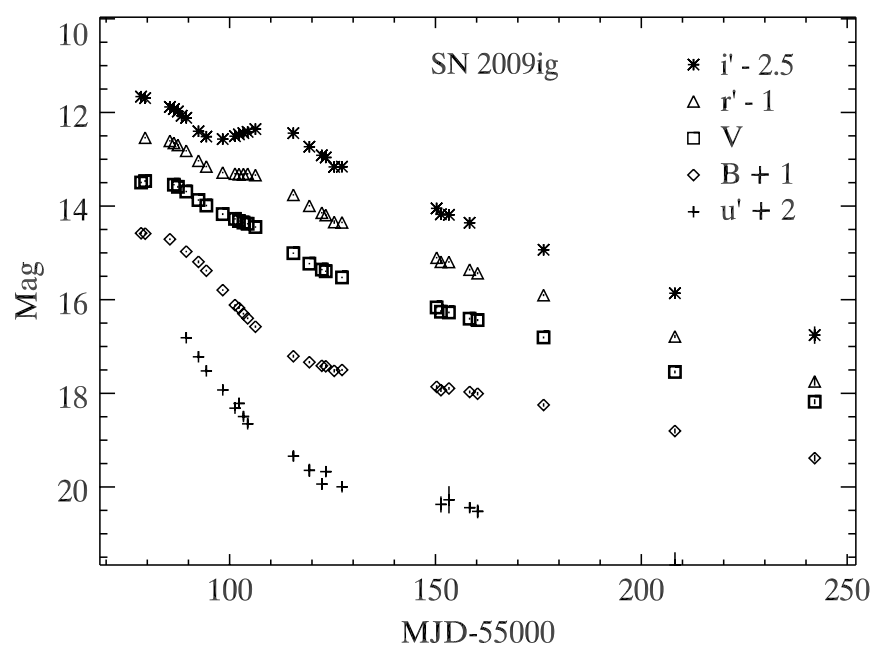

Figure 7. SN 2009ig, one of the better-sampled CfA4 light curves. The error bars are smaller than the symbols for most of the data points.

Web site. ${ }^{17}$ The print journal version and astro-ph version of Tables 4-6 only show a small portion of the full data set. The natural-system comparison star photometry is also available at our Web site. For the SN photometry, the number of successful subtractions from a given night that survived outlier rejection is listed. For example, if there were two data images and seven reference images and no photometry points were rejected, then $N=14$. Usually there was only one data image per night. The uncertainties that were added in quadrature to obtain the final uncertainty are listed. The $\mathrm{C} 12 B V r^{\prime} i^{\prime}$ passbands will be available soon. The natural-system passbands and photometry can be used together to avoid the uncertainty of using starderived color coefficients for $\mathrm{SNe}$.

We make no effort to estimate the additional uncertainties in the standard-system SN Ia photometry due to the lack of $s$-corrections but note that the uncertainties listed in Table 6 are certainly an underestimate. The natural-system uncertainties are the same as the standard-system uncertainties because we chose not to add the stastistical uncertainty of the color terms, which would increase the total uncertainty to about 1.005 times the natural-system values and are thus negligible. As evident in Table 5, our natural-system SN

\footnotetext{
17 http://www.cfa.harvard.edu/supernova/CfA4
} 
Table 4

Standard-system Comparison Star Photometry

\begin{tabular}{|c|c|c|c|c|c|c|c|c|c|c|c|c|c|c|c|c|c|c|}
\hline $\mathrm{SN}$ & Star & R.A. & Decl. & $V$ & $\sigma$ & $N$ & $U-B$ & $\sigma$ & $N$ & $B-V$ & $\sigma$ & $N$ & $V-r^{\prime}$ & $\sigma$ & $N$ & $V-i^{\prime}$ & $\sigma$ & $N$ \\
\hline 2010ai & 01 & $12: 59: 44.963$ & $+27: 56: 57.54$ & 15.365 & 0.015 & 3 & $\ldots$ & $\ldots$ & 0 & & 0.021 & 3 & 0.271 & 0.010 & 3 & 0.477 & 0.015 & 3 \\
\hline 2010ai & 02 & $12: 59: 41.382$ & $+28: 00: 07.92$ & 16.715 & 0.015 & 7 & 0.115 & 0.131 & 1 & 0.620 & 0.019 & & 0.185 & 0.010 & 7 & 0.345 & 0.012 & 7 \\
\hline 2010ai & 03 & $12: 59: 34.571$ & $+27: 54: 52.44$ & 17.505 & 0.013 & 7 & -0.823 & 0.174 & 1 & 0.077 & 0.020 & & -0.088 & 0.016 & 7 & -0.281 & 0.021 & 7 \\
\hline 2010ai & 04 & $12: 59: 28.781$ & $+27: 56: 15.51$ & 12.449 & 0.012 & 7 & $\ldots$ & $\ldots$ & 0 & 1.152 & 0.016 & 7 & $\ldots$ & $\ldots$ & 0 & $\ldots$ & $\ldots$ & 0 \\
\hline 2010ai & 05 & $12: 59: 26.528$ & $+28: 00: 24.01$ & 16.656 & 0.017 & 7 & 0.014 & 0.129 & 1 & 0.639 & 0.019 & 7 & 0.157 & 0.013 & 7 & 0.283 & 0.023 & 7 \\
\hline 2010ai & 06 & $12: 59: 25.270$ & $+27: 59: 07.64$ & 17.583 & 0.015 & 7 & & & 0 & & 0.029 & 7 & 0.145 & 0.012 & 7 & & 0.030 & 7 \\
\hline 2010ai & 07 & $12: 59: 24.761$ & $+27: 56: 24.16$ & 15.921 & 0.013 & 7 & & & 0 & & & 7 & 0.287 & & 7 & & 0.010 & 7 \\
\hline 2010ai & 08 & $12: 59: 18.650$ & $+28: 01: 43.34$ & 15.433 & 0.012 & 7 & & & 0 & 1.260 & 0.016 & 7 & 0.497 & 0.009 & 7 & 0.909 & 0.010 & 7 \\
\hline 2010ai & 09 & $12: 59: 15.882$ & $+27: 57: 10.68$ & 16.109 & 0.013 & 7 & -0.200 & 0.110 & 1 & 0.523 & 0.018 & r & 0.113 & 0.009 & 7 & 0.202 & 0.013 & 7 \\
\hline 2010ai & 10 & $12: 59: 13.739$ & $+28: 02: 10.48$ & 16.916 & 0.013 & 7 & -0.161 & 0.141 & 1 & 0.522 & 0.018 & 7 & 0.124 & 0.011 & 7 & 0.249 & 0.011 & 7 \\
\hline 2010ai & 11 & $12: 59: 11.788$ & $+28: 00: 04.02$ & 15.492 & 0.012 & 7 & 0.302 & 0.096 & 1 & 0.784 & 0.016 & 7 & 0.234 & 0.009 & 7 & 0.398 & 0.010 & 7 \\
\hline 2010ai & 12 & $12: 59: 04.168$ & $+28: 03: 48.67$ & 16.084 & 0.012 & 7 & 0.250 & 0.110 & 1 & 0.761 & 0.018 & 7 & 0.214 & 0.010 & 7 & 0.389 & 0.012 & 7 \\
\hline 2010ai & 13 & 12:59:01.189 & $+28: 02: 03.98$ & 15.265 & 0.013 & 7 & 0.416 & 0.091 & 1 & 0.941 & 0.017 & 7 & 0.293 & 0.010 & 7 & 0.557 & 0.010 & 7 \\
\hline
\end{tabular}

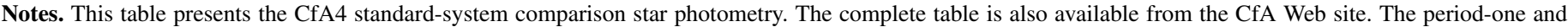

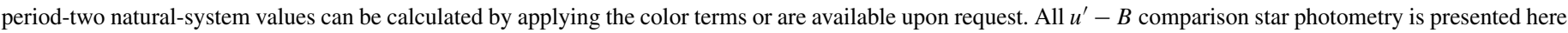

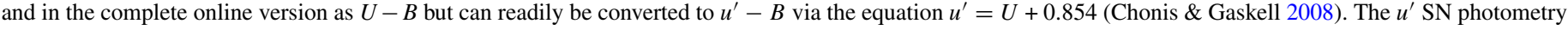
is presented as $u^{\prime}$ in Tables 5 and 6 .

(This table is available in its entirety in a machine-readable form in the online journal. A portion is shown here for guidance regarding its form and content.)

Table 5

Natural-system SN Photometry

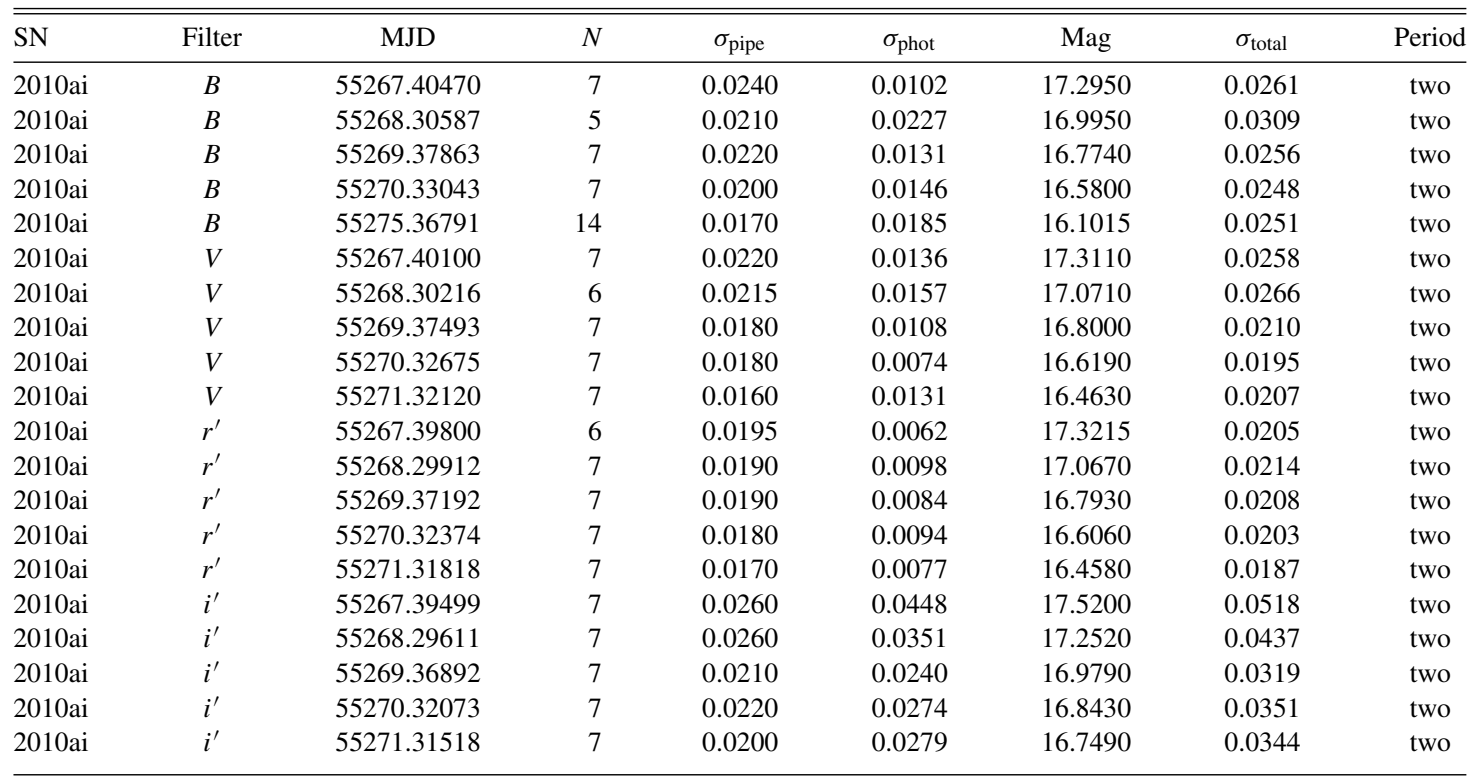

Notes. This table presents the CfA4 natural-system SN photometry. The number of successful subtractions from that night that survived outlier rejection is listed in the fourth column. For example, if there were two data images and seven reference images and none were rejected, then $N=14$. Usually there was only one data image per night. The median of the pipeline-generated uncertainties of the surviving photometry points is listed in the fifth column. The standard deviation of the surviving photometry values for that date is listed in the sixth column. These two values are added in quadrature to produce the total uncertainty. The last column lists what period the photometry belongs to and is of crucial importance so that the corresponding period-one or period-two passbands or color terms are used. Period one is before MJD $=55058$ and period two is after. Only the first five nights in each band of one SN are shown here. The complete table with all bands and all SNe is also available from the CfA Web site.

(This table is available in its entirety in a machine-readable form in the online journal. A portion is shown here for guidance regarding its form and content.)

photometry has a precision, $\sigma_{\text {total }}$, of $\lesssim 0.03 \mathrm{mag}$ in $B V r^{\prime} i^{\prime}$, $\lesssim 0.06 \mathrm{mag}$ in $u^{\prime}$, and $\lesssim 0.07 \mathrm{mag}$ in $U$ for points brighter than $17.5 \mathrm{mag}$.

We also estimate a systematic uncertainty in the naturalsystem photometry of each $\mathrm{SN}$ of $0.014,0.010,0.012,0.014$, 0.046 , and $0.073 \mathrm{mag}$ in $B V r^{\prime} i^{\prime} u^{\prime} U$, respectively. These systematic uncertainties are not included in our natural-system photometry uncertainties in Table 5. They are due to the uncertainties in the zero points of the photometric solution. They were derived by dividing the median uncertainty of all nights' photometric-solution zero points in a given band by the square root of the average number of nights of photometric calibration across all SNe in the same band. For example, the median uncertainty in the $V$ solution is $0.02 \mathrm{mag}$, and the average number 
Table 6

Standard-system SN Photometry

\begin{tabular}{lccccccc}
\hline \hline SN & Filter & MJD & $N$ & $\sigma_{\text {pipe }}$ & $\sigma_{\text {phot }}$ & Mag & $\sigma_{\text {total }}$ \\
\hline 2010ai & $B$ & 55267.40470 & 7 & 0.0240 & 0.0102 & 17.2932 & 0.0261 \\
2010ai & $B$ & 55268.30587 & 5 & 0.0210 & 0.0227 & 16.9861 & 0.0309 \\
2010ai & $B$ & 55269.37863 & 7 & 0.0220 & 0.0131 & 16.7710 & 0.0256 \\
2010ai & $B$ & 55270.33043 & 7 & 0.0200 & 0.0146 & 16.5755 & 0.0248 \\
2010ai & $B$ & 55275.36791 & 14 & 0.0170 & 0.0185 & 16.1004 & 0.0251 \\
2010ai & $V$ & 55267.40100 & 7 & 0.0220 & 0.0136 & 17.3114 & 0.0258 \\
2010ai & $V$ & 55268.30216 & 6 & 0.0215 & 0.0157 & 17.0730 & 0.0266 \\
2010ai & $V$ & 55269.37493 & 7 & 0.0180 & 0.0108 & 16.8007 & 0.0210 \\
2010ai & $V$ & 55270.32675 & 7 & 0.0180 & 0.0074 & 16.6200 & 0.0195 \\
2010ai & $V$ & 55271.32120 & 7 & 0.0160 & 0.0131 & 16.4644 & 0.0207 \\
2010ai & $r^{\prime}$ & 55267.39800 & 6 & 0.0195 & 0.0062 & 17.3216 & 0.0205 \\
2010ai & $r^{\prime}$ & 55268.29912 & 7 & 0.0190 & 0.0098 & 17.0691 & 0.0214 \\
2010ai & $r^{\prime}$ & 55269.37192 & 7 & 0.0190 & 0.0084 & 16.7939 & 0.0208 \\
2010ai & $r^{\prime}$ & 55270.32374 & 7 & 0.0180 & 0.0094 & 16.6074 & 0.0203 \\
2010ai & $r^{\prime}$ & 55271.31818 & 7 & 0.0170 & 0.0077 & 16.4595 & 0.0187 \\
2010ai & $i^{\prime}$ & 55267.39499 & 7 & 0.0260 & 0.0448 & 17.5155 & 0.0518 \\
2010ai & $i^{\prime}$ & 55268.29611 & 7 & 0.0260 & 0.0351 & 17.2498 & 0.0437 \\
2010ai & $i^{\prime}$ & 55269.36892 & 7 & 0.0210 & 0.0240 & 16.9755 & 0.0319 \\
2010ai & $i^{\prime}$ & 55270.32073 & 7 & 0.0220 & 0.0274 & 16.8388 & 0.0351 \\
2010ai & $i^{\prime}$ & 55271.31518 & 7 & 0.0200 & 0.0279 & 16.7437 & 0.0344 \\
\hline & & & & & & & \\
\end{tabular}

Notes. This table presents the CfA4 standard-system SN photometry. The number of successful subtractions from that night that survived outlier rejection is listed in the fourth column. For example, if there were two data images and seven reference images and none were rejected, then $N=14$. Usually there was only one data image per night. The median of the pipeline-generated uncertainties of the surviving photometry points is listed in the fifth column. The standard deviation of the surviving photometry values for that date is listed in the sixth column. These two values are added in quadrature to produce the total uncertainty. Only the first five nights in each band of one SN are shown here. The complete table with all bands and all SNe is also available from the CfA Web site.

(This table is available in its entirety in a machine-readable form in the online journal. A portion is shown here for guidance regarding its form and content.)

of nights is 3.6, resulting in $0.01 \mathrm{mag}$. These estimates are in rough agreement with the differences between samples seen in Table 7, which also serve as an estimate of the systematic offsets as explained below. The comparison star uncertainties in Table 4 contain this systematic uncertainty, but the photometry pipeline treats them as purely statistical, and so it gets lost due to the relatively large number of comparison stars used for each SN.

\section{PHOTOMETRY COMPARISON WITH OTHER SAMPLES}

Twelve CfA4 SN Ia light curves overlap with recent LOSS photometry (Ganeshalingam et al. 2010; Silverman et al. 2011), and eight overlap with CSP2. Comparisons between the three groups were made in the standard system. The LOSS and CSP2 comparison star photometry was published in the standard system, as was the LOSS SN photometry. The CSP2 SN photometry was only presented in the natural system. The CSP2 SN standard-system photometry (without $s$-corrections) was provided to us for the overlapping objects (M. Stritzinger 2011, private communication). We emphasize that none of the CfA4, LOSS, or CSP2 standard-system SN photometry is $s$-corrected. Since SN spectral energy distributions (SEDs) differ from the stellar SEDs used to derive the photometric transformation coefficients, the comparisons of the standard-system SN photometry here are limited to providing a reasonable but not highly accurate idea of the agreement between samples. A description of $s$-corrections and why they are needed for more accurate transformation to the standard system is given in Suntzeff (2000). An application of this method is presented in Stritzinger et al. (2002).

Table 7 shows the mean difference of all the comparison stars in common between CfA4, LOSS, and CSP2. There is relatively good agreement $(<0.015 \mathrm{mag})$ in all bands and between all samples except in $B$, where CSP2 and LOSS differ by $0.035 \mathrm{mag}$ and CSP2 and CfA 4 differ by $0.022 \mathrm{mag}$. The differences in the mean are larger than the standard error of the mean in all cases except for LOSS-CSP2 in $V$. The comparison star photometry does not require $s$-corrections and gives a good idea of the systematic offsets that would exist between the three groups' SN photometry after accurate $s$-corrections. The mean difference between two groups' photometry can be taken by itself as a good estimate of the systematic offset. Another approach, in the spirit of having $\chi^{2}=1$, is to add enough systematic uncertainty in quadrature to the statistical uncertainty so this total uncertainty is equal to the absolute value of the mean difference. This is only performed when the absolute value of the mean difference is greater than the statistical uncertainty. Following this second approach and assuming that the stars in common are representative of the whole samples suggests that LOSS is systematically brighter than CfA 4 by 0.008 mag in $B$ and fainter by $0.011 \mathrm{mag}$ in $V$. LOSS is 0.034 mag brighter than CSP 2 in $B$ and consistent in $V$. CSP2 would be 0.022 mag fainter than CfA4 in $B$ and 0.015 mag fainter in $V$. However, it should be noted that there are only $\sim 80$ stars in common between LOSS and CfA 4 and between CSP 2 and CfA 4 , and only $~ 45$ between LOSS and CSP2. Histograms that show all of the comparison star differences are presented in Figures 8-10. The distributions are reasonably symmetric around their mean except for the $B$ LOSS-CSP2 histogram.

In order to compare the SN photometry, a cubic spline was fit to the light curve from one group (for descriptive purposes here, group A). The spline was always of the same order. It was allowed to extend one day beyond the earliest and latest

Table 7

Comparison Star Mean Differences

\begin{tabular}{lrccccccc}
\hline \hline SN Samples & \multicolumn{1}{c}{$\mu_{\Delta B}$} & $\sigma / \sqrt{N}$ & $\sigma_{\Delta B}$ & $N_{\text {stars }}$ & $\mu_{\Delta V}$ & $\sigma / \sqrt{N}$ & $\sigma_{\Delta V}$ & $N_{\text {stars }}$ \\
\hline LOSS-CfA4 & -0.0087 & 0.0037 & 0.0346 & 86 & 0.0109 & 0.0028 & 0.0263 & 86 \\
LOSS-CSP2 & -0.0346 & 0.0071 & 0.0489 & 48 & -0.0016 & 0.0048 & 0.0319 & 44 \\
CSP2-CfA4 & 0.0223 & 0.0032 & 0.0289 & 81 & 0.0149 & 0.0025 & 0.0217 & 77 \\
\hline & $\mu_{\Delta r^{\prime}}$ & $\sigma / \sqrt{N}$ & $\sigma_{\Delta r^{\prime}}$ & $N_{\text {stars }}$ & $\mu_{\Delta i^{\prime}}$ & $\sigma / \sqrt{N}$ & $\sigma_{\Delta i^{\prime}}$ & $N_{\text {stars }}$ \\
\hline CSP2-CfA4 & 0.0000 & 0.0033 & 0.0300 & 79 & -0.0059 & 0.0027 & 0.0236 & 78 \\
\hline
\end{tabular}

Notes. Listed are the mean, standard error of the mean, and standard deviation of the differences in the photometry of all of the comparison stars in common between CfA4, LOSS, and CSP2. Also provided is the number of comparison stars in common. 

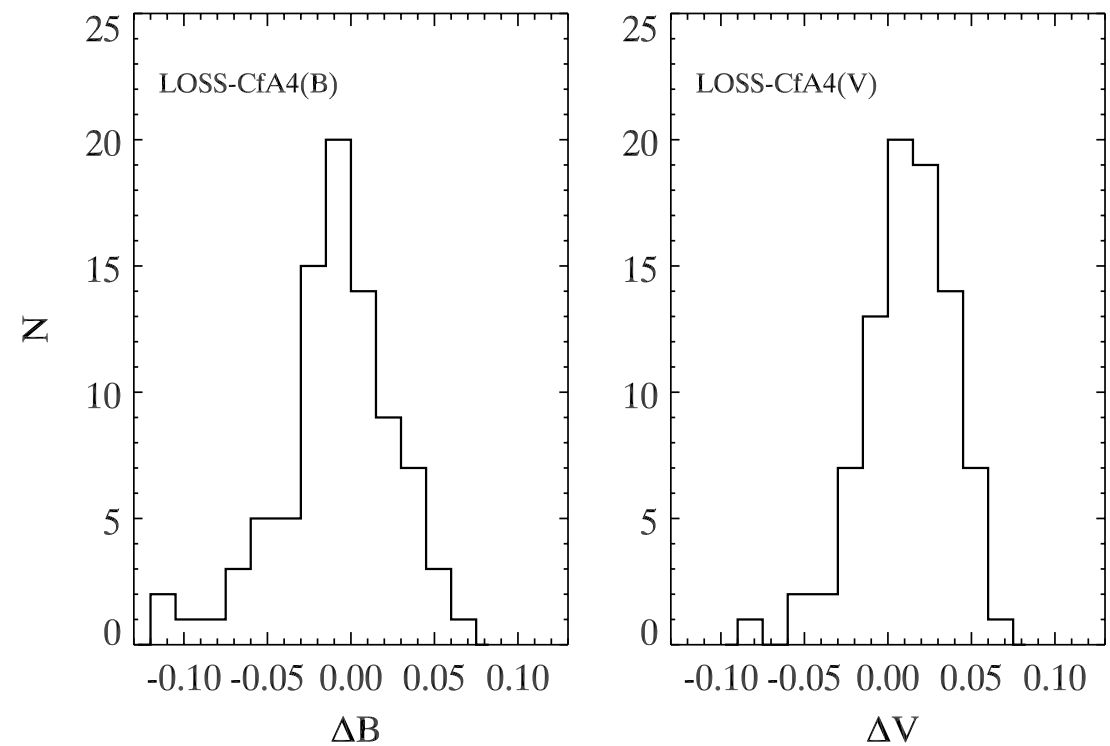

Figure 8. Histograms of the differences in the LOSS-CfA4 $B V$ photometry for all the comparison stars in common between the two samples. The distributions are roughly symmetric.
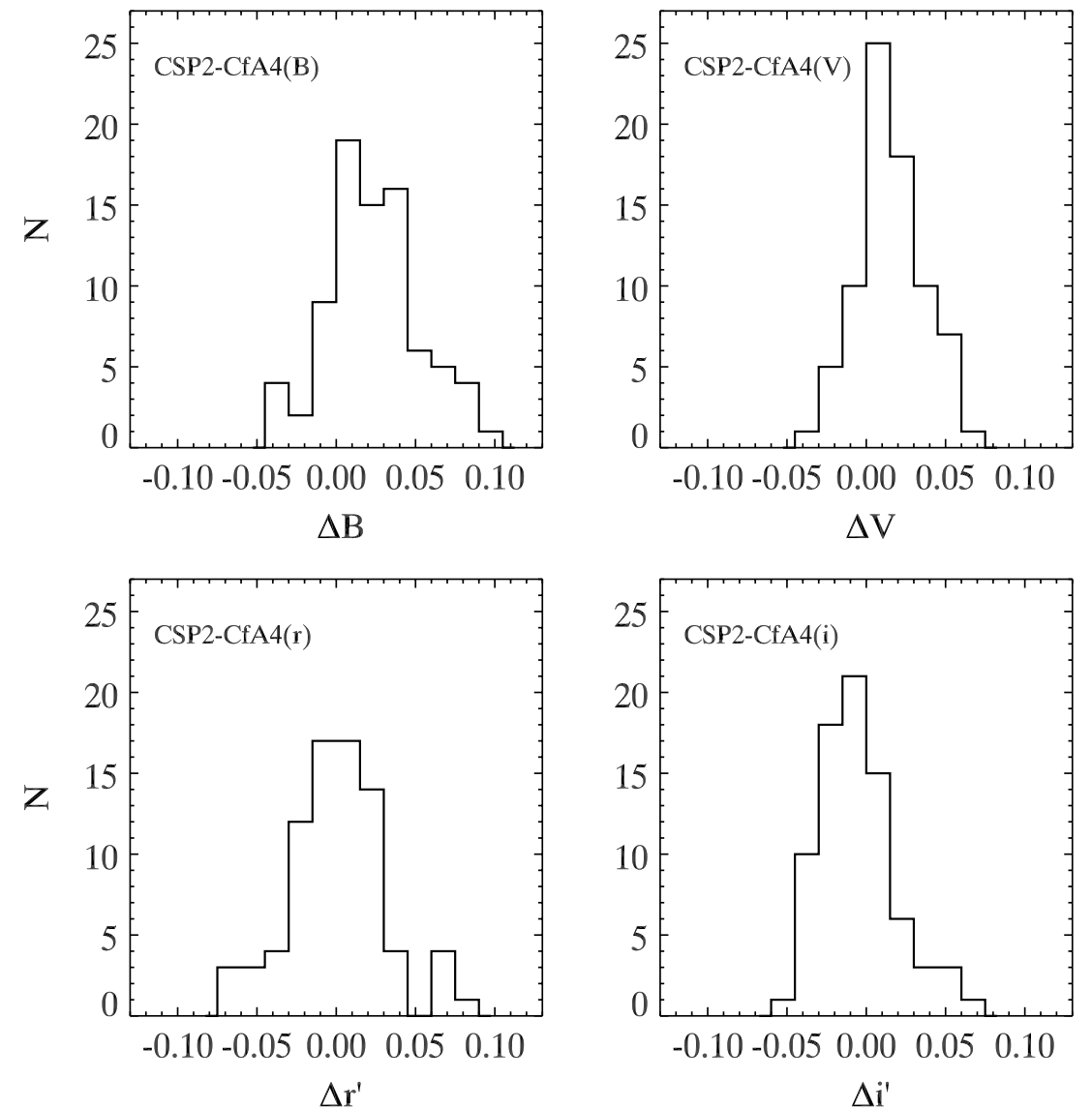

Figure 9. Histograms of the differences in the CSP2-CfA4 $B V r^{\prime} i^{\prime}$ photometry for all the comparison stars in common between the two samples. The distributions are roughly symmetric in $V r^{\prime}$ with slightly larger tails on the positive side in $B i^{\prime}$.

points. It was visually inspected to ensure that it smoothly fit the data. This spline was then subtracted from the other group's points (group B) that were within the spline's date range and had at least one data point from group A within four days. For each SN that we compared we fit a spline to each group's photometry and subtracted the other group's points. In cases where the light curves of each group are roughly equally well sampled and smooth, there is virtually no difference between which group's data are used for the spline. In these cases we presented the subtraction direction that gave rise to a slightly smaller reduced $\chi^{2}$. In cases where one group's light curve is more densely sampled and/or smooth, a superior spline fit was produced and we used that one to perform the comparison. In light of the inherent limitations of comparing non-s-corrected 

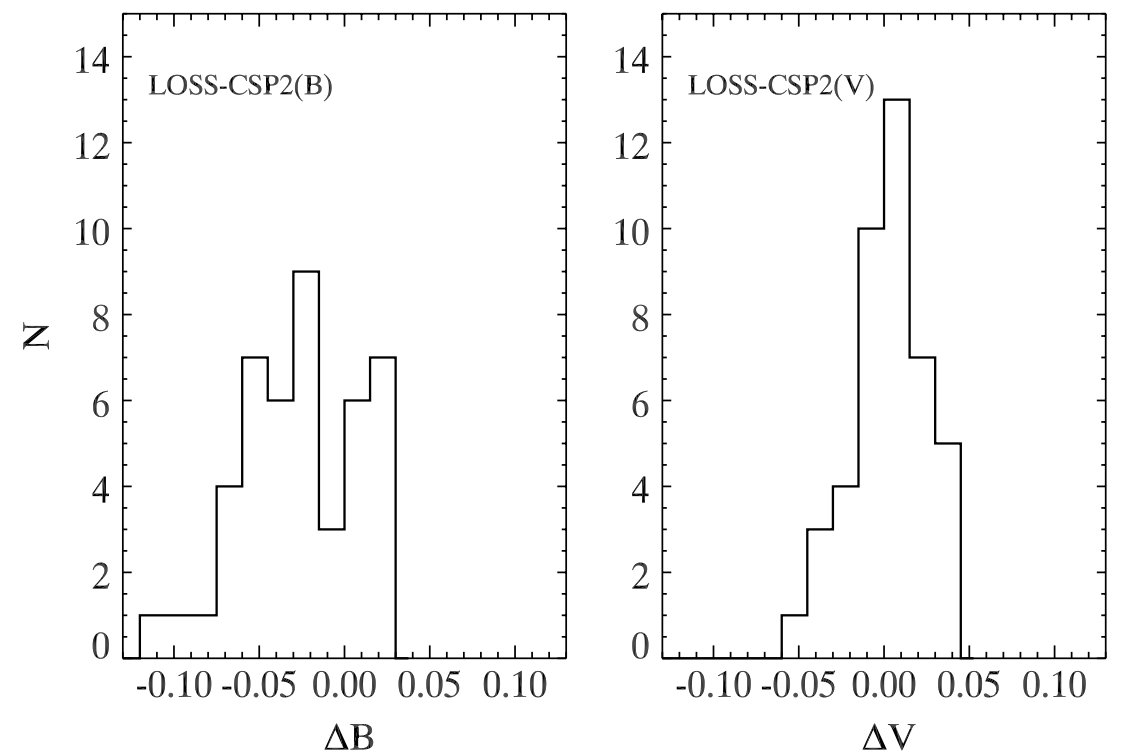

Figure 10. Histograms of the differences in the LOSS-CSP2 $B V$ photometry for all the comparison stars in common between the two samples. The distributions are roughly symmetric in $V$ but not in $B$.
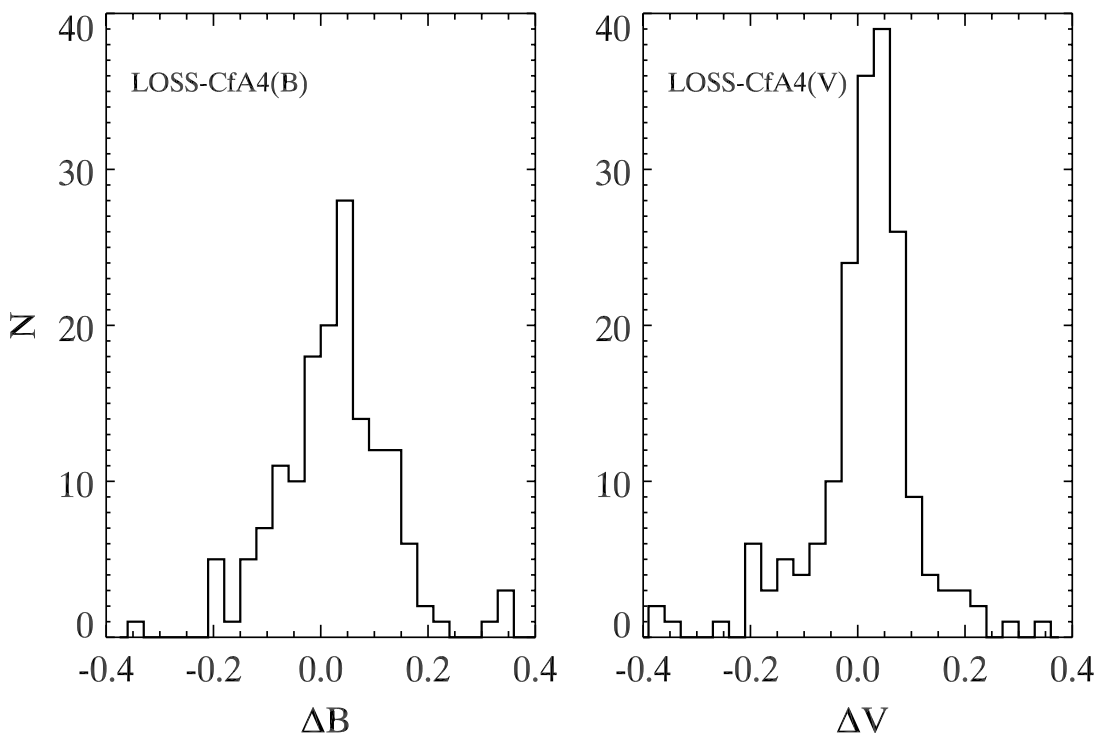

Figure 11. Histograms of the $B V$ differences of the LOSS-CfA4 SN photometry for the $12 \mathrm{SNe}$ in common between the two samples. The distributions are roughly symmetric.

Table 8

Sample-to-Sample SN Photometry Comparisons

\begin{tabular}{lcccccccc}
\hline \hline SN Samples & $\mu_{\Delta B}$ & $\chi_{v}^{2}(B)$ & $\sigma(B)$ & $\sigma_{<18}(B)$ & $\mu_{\Delta V}$ & $\chi_{v}^{2}(V)$ & $\sigma(V)$ & $\sigma_{<18}(V)$ \\
\hline LOSS-CfA4 & 0.036 & 3.24 & 0.110 & 0.075 & 0.035 & 3.50 & 0.099 & 0.070 \\
CSP2-CfA4 & 0.027 & 1.82 & 0.126 & 0.035 & 0.026 & 3.51 & 0.095 & 0.040 \\
\hline & $\mu_{\Delta r^{\prime}}$ & $\chi_{v}^{2}\left(r^{\prime}\right)$ & $\sigma\left(r^{\prime}\right)$ & $\sigma_{<18}\left(r^{\prime}\right)$ & $\mu_{\Delta i^{\prime}}$ & $\chi_{v}^{2}\left(i^{\prime}\right)$ & $\sigma\left(i^{\prime}\right)$ & $\sigma_{<18}\left(i^{\prime}\right)$ \\
\hline CSP2-CfA4 & -0.019 & 3.36 & 0.096 & 0.054 & 0.004 & 2.37 & 0.092 & 0.048 \\
\hline
\end{tabular}

Notes. The weighted mean, reduced $\chi^{2}$, and standard deviation of all subtractions of the SN photometry in common between CfA4, LOSS, and CSP2. Also, the standard deviation with points fainter than 18 mag is removed.

photometry, which only allows for a reasonable comparison, we opted not to do the slightly more involved task of combining both subtraction directions.

The weighted mean of the differences of all of the subtracted points between two groups in a given band is presented in Table 8. There is general agreement between CfA4, LOSS, and
CSP2 in these weighted means. CfA4 is $\sim 0.03$ mag brighter than CSP2 and LOSS in $B V, \sim 0.02$ mag fainter than CSP2 in $r^{\prime}$, and 0.004 mag brighter than CSP2 in $i^{\prime}$. The reduced $\chi^{2}$ ranges from 1.8 to 3.6. These should be understood as a modified reduced $\chi^{2}$ since the spline's degrees of freedom were not included in the calculation. The standard deviation of the 
Table 9

Comparison Star and SN Photometry Comparison

\begin{tabular}{|c|c|c|c|c|c|c|c|c|c|c|c|c|}
\hline Samples/SN & $\mu_{\Delta}$ & $\sigma / \sqrt{N}$ & $\sigma_{\Delta}$ & $N_{\text {stars }}$ & $\mu_{\Delta(\mathrm{wgt})}$ & $\chi_{v}^{2}$ & $\mu_{\Delta}$ & $\sigma / \sqrt{N}$ & $\sigma_{\Delta}$ & $N_{\text {stars }}$ & $\mu_{\Delta(\mathrm{wgt})}$ & $\chi_{v}^{2}$ \\
\hline LOSS-CfA4 & $B_{\text {stars }}$ & & & & $B_{\mathrm{SN}}$ & & $V_{\text {stars }}$ & & & & $V_{\mathrm{SN}}$ & \\
\hline $2007 b j$ & 0.014 & 0.009 & 0.028 & 10 & 0.0267 & 2.126 & 0.019 & 0.007 & 0.021 & 10 & 0.0500 & 5.996 \\
\hline 2007hj & 0.012 & 0.024 & 0.058 & 6 & 0.0438 & 3.676 & 0.019 & 0.014 & 0.035 & 6 & 0.0438 & 3.550 \\
\hline 2007le & -0.061 & 0.050 & 0.071 & 2 & 0.0172 & 3.127 & -0.032 & 0.052 & 0.074 & 2 & 0.0258 & 2.814 \\
\hline $2007 u x$ & 0.018 & 0.007 & 0.016 & 5 & -0.0788 & 1.454 & 0.014 & 0.008 & 0.018 & 5 & 0.0451 & 3.148 \\
\hline $2008 \mathrm{~A}$ & -0.021 & 0.011 & 0.043 & 14 & 0.0568 & 2.510 & 0.009 & 0.007 & 0.028 & 14 & 0.0367 & 7.460 \\
\hline $2008 \mathrm{C}$ & -0.022 & 0.006 & 0.027 & 23 & -0.0133 & 0.995 & 0.017 & 0.004 & 0.021 & 23 & 0.0295 & 1.184 \\
\hline 2008Q & -0.028 & 0.006 & 0.011 & 3 & 0.1327 & 55.403 & -0.046 & 0.005 & 0.008 & 3 & -0.0013 & 0.408 \\
\hline $2008 Z$ & 0.008 & 0.007 & 0.016 & 5 & -0.0173 & 0.659 & 0.012 & 0.008 & 0.019 & 5 & 0.0278 & 5.087 \\
\hline 2008ar & -0.002 & 0.004 & 0.005 & 2 & 0.0537 & 3.690 & -0.010 & 0.003 & 0.004 & 2 & 0.0126 & 4.183 \\
\hline $2008 \mathrm{dr}$ & -0.014 & 0.006 & 0.011 & 3 & 0.1129 & 0.647 & 0.034 & 0.006 & 0.011 & 3 & 0.0115 & 0.527 \\
\hline $2008 \mathrm{dt}$ & -0.001 & 0.008 & 0.022 & 8 & -0.0912 & 0.565 & 0.008 & 0.006 & 0.016 & 8 & -0.1304 & 4.058 \\
\hline $2008 \mathrm{ec}$ & -0.016 & $\ldots$ & $\ldots$ & 1 & $\ldots$ & $\ldots$ & -0.009 & $\ldots$ & $\ldots$ & 1 & $\ldots$ & $\ldots$ \\
\hline $2009 \mathrm{dc}$ & -0.002 & 0.007 & 0.015 & 4 & 0.0593 & 3.314 & 0.013 & 0.007 & 0.014 & 4 & 0.0497 & 3.230 \\
\hline CSP2-CfA4 & $B_{\text {stars }}$ & & & & $B_{\mathrm{SN}}$ & & $V_{\text {stars }}$ & & & & $V_{\mathrm{SN}}$ & \\
\hline 2007A & 0.016 & 0.009 & 0.024 & 7 & 0.0156 & 2.880 & 0.005 & 0.005 & 0.011 & 5 & 0.0113 & 4.647 \\
\hline 2007if & 0.024 & 0.011 & 0.028 & 7 & 0.0102 & 0.819 & 0.017 & 0.005 & 0.014 & 7 & 0.0946 & 3.424 \\
\hline 2007jg & 0.011 & 0.004 & 0.017 & 15 & 0.0577 & 1.167 & -0.004 & 0.005 & 0.020 & 14 & 0.0039 & 1.462 \\
\hline 2007le & 0.053 & 0.008 & 0.025 & 9 & 0.0346 & 4.573 & 0.042 & 0.006 & 0.017 & 9 & 0.0527 & 10.25 \\
\hline $2007 \mathrm{nq}$ & 0.035 & 0.002 & 0.005 & 5 & 0.0272 & 0.876 & 0.023 & 0.005 & 0.011 & 5 & 0.0150 & 1.637 \\
\hline $2008 \mathrm{C}$ & 0.005 & 0.003 & 0.013 & 15 & 0.0326 & 2.052 & 0.028 & 0.004 & 0.014 & 15 & 0.0227 & 4.184 \\
\hline 2008hv & 0.006 & 0.010 & 0.033 & 12 & 0.0033 & 1.387 & 0.005 & 0.006 & 0.020 & 12 & -0.0004 & 1.445 \\
\hline $2009 \mathrm{dc}$ & 0.052 & 0.007 & 0.024 & 11 & 0.0498 & 2.364 & 0.011 & 0.007 & 0.021 & 10 & 0.0221 & 1.979 \\
\hline CSP2-CfA4 & $r_{\text {stars }}^{\prime}$ & & & & $r_{\mathrm{SN}}^{\prime}$ & & $i_{\text {stars }}^{\prime}$ & & & & $i_{\mathrm{SN}}^{\prime}$ & \\
\hline 2007A & 0.035 & 0.012 & 0.032 & 7 & 0.0034 & 1.984 & 0.023 & 0.009 & 0.024 & 7 & 0.0108 & 0.375 \\
\hline 2007if & 0.005 & 0.008 & 0.021 & 7 & 0.0345 & 1.196 & -0.001 & 0.008 & 0.020 & 7 & 0.0301 & 2.220 \\
\hline 2007jg & -0.006 & 0.004 & 0.016 & 14 & 0.0014 & 1.352 & -0.015 & 0.005 & 0.017 & 13 & 0.1378 & 3.892 \\
\hline 2007le & 0.036 & 0.008 & 0.023 & 9 & 0.0077 & 1.005 & 0.023 & 0.008 & 0.024 & 9 & 0.0218 & 2.979 \\
\hline $2007 \mathrm{nq}$ & 0.016 & 0.005 & 0.012 & 5 & -0.0286 & 1.056 & -0.004 & 0.007 & 0.015 & 5 & 0.0281 & 1.063 \\
\hline $2008 C$ & -0.006 & 0.003 & 0.012 & 15 & -0.0353 & 7.983 & -0.026 & 0.002 & 0.009 & 15 & -0.0369 & 4.207 \\
\hline $2008 \mathrm{hv}$ & -0.003 & 0.005 & 0.016 & 12 & -0.0460 & 5.842 & -0.008 & 0.004 & 0.015 & 12 & -0.0282 & 3.254 \\
\hline $2009 \mathrm{dc}$ & -0.047 & 0.007 & 0.021 & 10 & -0.0672 & 7.882 & -0.018 & 0.007 & 0.022 & 10 & -0.0021 & 0.527 \\
\hline LOSS-CSP2 & $B_{\text {stars }}$ & & & & $B_{\mathrm{SN}}$ & & $V_{\text {stars }}$ & & & & $V_{\mathrm{SN}}$ & \\
\hline $2006 \mathrm{bt}$ & 0.004 & 0.010 & 0.027 & 7 & $\ldots$ & $\ldots$ & 0.011 & 0.008 & 0.020 & 7 & $\ldots$ & $\ldots$ \\
\hline 2006еj & 0.016 & $\ldots$ & $\ldots$ & 1 & $\ldots$ & $\ldots$ & $\ldots$ & $\ldots$ & $\ldots$ & 0 & $\ldots$ & \\
\hline $2006 \mathrm{hb}$ & 0.010 & 0.007 & 0.015 & 5 & $\ldots$ & $\ldots$ & 0.025 & 0.009 & 0.018 & 4 & $\ldots$ & $\ldots$ \\
\hline 2007af & -0.038 & 0.004 & 0.009 & 4 & $\ldots$ & $\ldots$ & 0.012 & 0.004 & 0.009 & 4 & $\ldots$ & $\ldots$ \\
\hline $2007 \mathrm{bc}$ & -0.107 & 0.040 & 0.069 & 3 & $\ldots$ & $\ldots$ & -0.003 & 0.009 & 0.013 & 2 & $\ldots$ & $\ldots$ \\
\hline $2007 \mathrm{ca}$ & -0.058 & 0.013 & 0.028 & 5 & $\ldots$ & $\ldots$ & -0.036 & 0.006 & 0.013 & 5 & $\ldots$ & $\ldots$ \\
\hline 2007le & -0.109 & 0.032 & 0.064 & 4 & 0.0029 & 2.364 & -0.039 & 0.036 & 0.072 & 4 & -0.0343 & 6.137 \\
\hline $2008 \mathrm{C}$ & -0.018 & 0.008 & 0.025 & 11 & -0.0198 & 3.314 & -0.007 & 0.005 & 0.018 & 11 & 0.0021 & 1.410 \\
\hline $2009 \mathrm{dc}$ & -0.044 & 0.012 & 0.034 & 8 & 0.0030 & 0.903 & 0.017 & 0.006 & 0.017 & 7 & 0.0369 & 3.767 \\
\hline
\end{tabular}

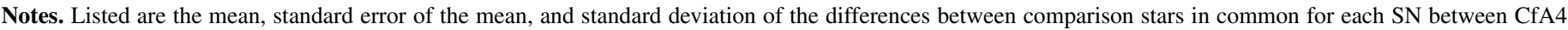

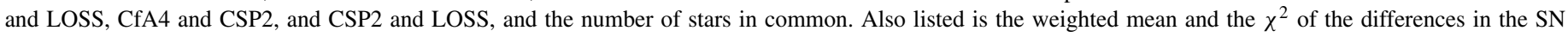
photometry.

differences is $\sim 0.1 \mathrm{mag}$. This is reduced to $0.04-0.05 \mathrm{mag}$ for the CSP2-CfA4 comparison when points fainter than $18 \mathrm{mag}$ are removed and to 0.07 mag for LOSS-CfA4. A comparison of LOSS versus CSP objects was only performed on the three SNe that were also in common with CfA4. Histograms of the LOSS-CfA4 and CSP2-CfA4 differences for all the subtracted points are shown in each filter in Figures 11 and 12, respectively. The distributions of the differences are all roughly symmetric. The lack of $s$-corrections, to take into account the response of different detectors to SN Ia SEDs, makes the SN photometry comparisons less accurate but still gives a reasonable estimate of how well they agree. Accurate $s$-corrections may resolve some of the discrepancies.
Table 9 presents the comparisons of the individual $\mathrm{SNe}$, showing the mean difference and number of comparison stars in common, as well as the weighted mean and reduced $\chi^{2}$ of the SN photometry differences. There seems to be very little correlation between the differences in the comparison star and SN photometry between LOSS and CfA4, but there does seem to be between CSP2 and CfA4.

The comparisons of the three SNe Ia in common between CfA4, LOSS, and CSP2 are shown in Table 10. For SN 2007le, the best agreement in $V$ is between LOSS and CfA4 and in $B$ is between LOSS and CSP2. For SN 2008C, the best agreement in $V$ is between LOSS and CSP 2 and in $B$ is between LOSS and CfA4. Finally, for SN 2009dc, the best agreement 

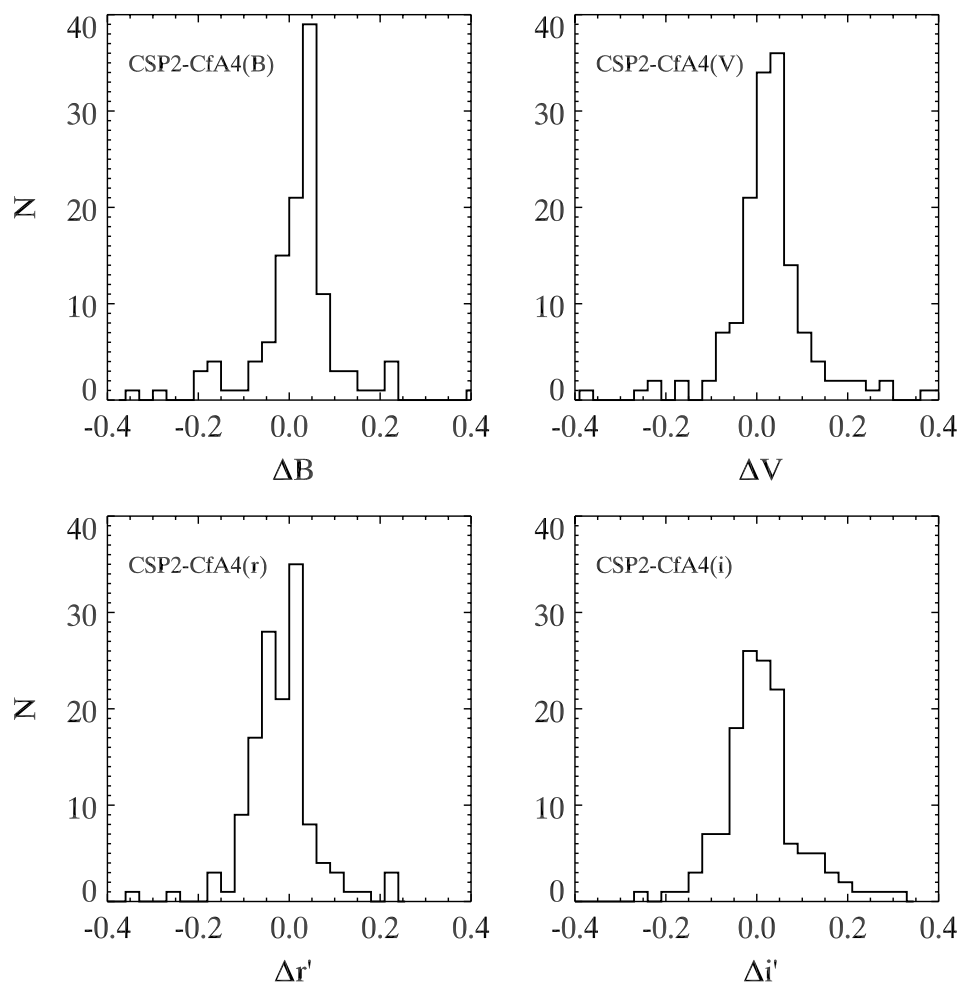

Figure 12. Histograms of the $B V r^{\prime} i^{\prime}$ differences of the CSP2-CfA4 SN photometry for the eight $\mathrm{SNe}$ in common between the two samples. The distributions are roughly symmetric.

Table 10

Comparing the Three SNe in Common

\begin{tabular}{|c|c|c|c|c|c|c|c|}
\hline SN/Samples & $\mu_{\Delta}$ & $\sigma / \sqrt{N}$ & $\sigma_{\Delta}$ & $N_{\text {stars }}$ & $\mu_{\Delta(\mathrm{wgt})}$ & $\sigma$ & $\chi_{v}^{2}$ \\
\hline 2007le & $B_{\text {stars }}$ & & & & $B_{\mathrm{SN}}$ & & \\
\hline CSP2-CfA4 & 0.053 & 0.008 & 0.025 & 9 & 0.0346 & 0.0340 & 4.573 \\
\hline LOSS-CfA4 & -0.061 & 0.050 & 0.071 & 2 & 0.0172 & 0.0521 & 3.127 \\
\hline LOSS-CSP2 & -0.109 & 0.032 & 0.064 & 4 & 0.0029 & 0.0434 & 4.204 \\
\hline $2008 \mathrm{C}$ & $B_{\text {stars }}$ & & & & $B_{\mathrm{SN}}$ & & \\
\hline CSP2-CfA4 & 0.005 & 0.003 & 0.013 & 15 & 0.0326 & 0.1976 & 2.052 \\
\hline LOSS-CfA4 & -0.022 & 0.006 & 0.027 & 23 & -0.0133 & 0.0742 & 0.995 \\
\hline LOSS-CSP2 & -0.018 & 0.008 & 0.025 & 11 & -0.0198 & 0.0226 & 1.565 \\
\hline $2009 \mathrm{dc}$ & $B_{\text {stars }}$ & & & & $B_{\mathrm{SN}}$ & & \\
\hline CSP2-CfA4 & 0.052 & 0.007 & 0.024 & 11 & 0.0498 & 0.0219 & 2.364 \\
\hline LOSS-CfA4 & -0.002 & 0.007 & 0.015 & 4 & 0.0593 & 0.0715 & 3.314 \\
\hline LOSS-CSP2 & -0.044 & 0.012 & 0.034 & 8 & 0.0030 & 0.0213 & 0.903 \\
\hline 2007le & $V_{\text {stars }}$ & & & & $V_{\mathrm{SN}}$ & & \\
\hline CSP2-CfA4 & 0.042 & 0.006 & 0.017 & 9 & 0.0527 & 0.0201 & 10.254 \\
\hline LOSS-CfA4 & -0.032 & 0.052 & 0.074 & 2 & 0.0258 & 0.0385 & 2.814 \\
\hline LOSS-CSP2 & -0.039 & 0.036 & 0.072 & 4 & -0.0343 & 0.0464 & 6.137 \\
\hline $2008 \mathrm{C}$ & $V_{\text {stars }}$ & & & & $V_{\mathrm{SN}}$ & & \\
\hline CSP2-CfA4 & 0.028 & 0.004 & 0.014 & 15 & 0.0227 & 0.0771 & 4.184 \\
\hline LOSS-CfA4 & 0.017 & 0.004 & 0.021 & 23 & 0.0295 & 0.0303 & 1.184 \\
\hline LOSS-CSP2 & -0.007 & 0.005 & 0.018 & 11 & 0.0021 & 0.0218 & 1.410 \\
\hline $2009 \mathrm{dc}$ & $V_{\text {stars }}$ & & & & $V_{\mathrm{SN}}$ & & \\
\hline CSP2-CfA4 & 0.011 & 0.007 & 0.021 & 10 & 0.0221 & 0.0168 & 1.979 \\
\hline LOSS-CfA4 & 0.013 & 0.007 & 0.014 & 4 & 0.0497 & 0.0578 & 3.230 \\
\hline LOSS-CSP2 & 0.017 & 0.006 & 0.017 & 7 & 0.0369 & 0.0191 & 3.767 \\
\hline
\end{tabular}

Notes. The mean difference, standard error of the mean, and standard deviation of the comparison star photometry and number of stars in common for each SN Ia are presented in Columns 2 through 5. The weighted mean, standard deviation, and reduced $\chi^{2}$ of the differences in the SN photometry are presented in the final three columns. in $V$ is between CSP2 and CfA4 and in $B$ is between LOSS and CSP2.

The main message from these comparisons is that the three groups are in reasonable agreement but that systematic uncertainties and effects are present in both the comparison stars and the SN photometry. A more definitive comparison of the SN photometry would require accurate $s$-corrections.

The primary goal of SN Ia photometry is to produce accurate distances for cosmological purposes. K. Mandel et al. (2012, in preparation) will provide an in-depth analysis of nearby distances that will include all recently published nearby optical and NIR photometry. Part of this will examine offsets between different samples.

\section{CONCLUSION}

The CfA4 sample consists of 94 nearby SN Ia optical light curves. Most of these are new objects, while 17 of them were also observed by LOSS or CSP2 and have adequate agreement between the different groups. The CfA4 sample is presented in both standard and natural systems. Each of our 94 SN Ia data images had at least one reference image subtracted. In most cases, we had multiple reference images, leading to improved knowledge of the net flux and of its uncertainty. CfA4 is the first large nearby sample to have its natural-system passbands determined by use of a tunable laser and calibrated photodiode (C. Cramer et al. 2012, in preparation). Deposits/condensation on the camera likely caused there to be two time periods with different average $B-V$ and $V-r^{\prime}$ color coefficients and naturalsystem passbands. However, the separation of the photometry, calibration, and natural-system passbands into the two time periods takes care of this problem.

Systematic uncertainties are now the largest obstacle in improving understanding of the expansion history of the universe. One of these systematic uncertainties is in the SN Ia photometry 
itself. Ensuring stable instruments and understanding the detector passbands involved are critical. In the case of CfA4 the deposits/condensation shifted the passbands, but careful calibrations-both the standard star observations and the C12 passband measurements-enabled this to be understood and overcome.

We thank the staff at FLWO for their dedicated work in maintaining the $1.2 \mathrm{~m}$ telescope and instruments. We also thank M. Stritzinger, W. Li, and M. Ganeshalingam for help in comparing the CfA4 sample with the CSP2 and LOSS samples. Finally, we appreciate discussions with K. Mandel. This work has been supported, in part, by NSF grants AST0606772 and AST0907903 to Harvard University.

Facility: FLWO:1.2m

\section{REFERENCES}

Alard, C. 2000, A\&AS, 144, 363

Alard, C., \& Lupton, R. 1998, ApJ, 503, 325

Aldering, G., Adam, G., Antilogus, P., et al. 2002, Proc. SPIE, 4836, 61

Amanullah, R., Lidman, C., Rubin, D., et al. 2010, ApJ, 716, 712

Bailey, S., Aldering, G., Antilogus, P., et al. 2009, A\&A, 500, L17

Becker, A. C., Wittman, D. M., Boeshaar, P. C., et al. 2004, ApJ, 611, 418

Bertin, E., Mellier, Y., Radovich, M., et al. 2002, in ASP Conf. Ser. 281, Astronomical Data Analysis Software and Systems XI, ed. D. A. Bohlender, D. Durand, \& T. H. Handley (San Francisco, CA: ASP), 228

Blondin, S., Mandel, K. S., \& Kirshner, R. P. 2011, A\&A, 526, 81

Blondin, S., Matheson, T., Kirshner, R. P., et al. 2012, AJ, 143, 126

Bongard, S., Baron, E., Smadja, G., Branch, D., \& Hauschildt, P. H. 2006, ApJ, 647,513

Chonis, T. S., \& Gaskell, C. M. 2008, AJ, 135, 264

Conley, A., Sullivan, M., Hsiao, E. Y., et al. 2008, ApJ, 681, 482

Contreras, C., Hamuy, M., Phillips, M. M., et al. 2010, AJ, 139, 519

Elias-Rosa, N., Benetti, S., Turatto, M., et al. 2008, MNRAS, 384, 107

Fabricant, D., Cheimets, P., Caldwell, N., \& Geary, J. 1998, PASP, 110, 79

Ganeshalingam, M., Li, W., Filippenko, A. V., et al. 2010, ApJS, 190, 418

Garg, A., Stubbs, C. W., Challis, P., et al. 2007, AJ, 133, 403

Guy, J., Astier, P., Baumont, S., et al. 2007, A\&A, 466, 11

Hachinger, S., Mazzali, P. A., Tanaka, M., Hillebrandt, W., \& Benetti, S. 2008, MNRAS, 389, 1087

Hamuy, M., Phillips, M. M., Suntzeff, N. B., et al. 1996a, AJ, 112, 2391

Hamuy, M., Phillips, M. M., Suntzeff, N. B., et al. 1996b, AJ, 112, 2408

Hicken, M., Challis, P., Jha, S., et al. 2009a, ApJ, 700, 331

Hicken, M., Wood-Vasey, W. M., Blondin, S., et al. 2009b, ApJ, 700, 1097

Holtzman, J. A., Marriner, J., Kessler, R., et al. 2008, AJ, 136, 2306

Jha, S., Kirshner, R. P., Challis, P., et al. 2006, AJ, 131, 527

Jha, S., Riess, A. G., \& Kirshner, R. P. 2007, ApJ, 659, 122
Kelly, P. L., Hicken, M., Burke, D. L., Mandel, K. S., \& Kirshner, R. P. 2010, ApJ, 715,743

Kirshner, R. P. 2010, in Dark Energy: Observational and Theoretical Approaches, ed. P. Ruiz-Lapuente (Cambridge: Cambridge Univ. Press), 151

Kowalski, M., Rubin, D., Aldering, G., et al. 2008, ApJ, 686, 749

Krisciunas, K., Hastings, N. C., Loomis, K., et al. 2000, ApJ, 539, 658

Krisciunas, K., Phillips, M. M., Stubbs, C., et al. 2001, AJ, 122, 1616

Krisciunas, K., Phillips, M. M., \& Suntzeff, N. B. 2004a, ApJ, 602, L81

Krisciunas, K., Phillips, M. M., Suntzeff, N. B., et al. 2004b, AJ, 127, 1664

Krisciunas, K., Prieto, J. L., Garnavich, P. M., et al. 2006, AJ, 131, 1639

Krisciunas, K., Suntzeff, N. B., Candia, P., et al. 2003, AJ, 125, 166

Krisciunas, K., Suntzeff, N. B., Phillips, M. M., et al. 2004c, AJ, 128, 3034

Lampeitl, H., Smith, M., Nichol, R. C., et al. 2010, ApJ, 722, 566

Landolt, A. U. 1992, AJ, 104, 372

Mandel, K., Narayan, G., \& Kirshner, R. P. 2011, ApJ, 731, 120

Matheson, T., Kirshner, R. P., Challis, P., et al. 2008, AJ, 135, 1598

Miknaitis, G., Pignata, G., Rest, A., et al. 2007, ApJ, 666, 674

Monet, D. 1998, USNO-A2.0 (11 CD-ROMs; Flagstaff, U.S. Naval Observatory)

Monet, D., Levine, S. E., Canzian, B., et al. 2003, AJ, 125, 984

Nugent, P., Phillips, M., Baron, E., Branch, D., \& Hauschildt, P. 1995, ApJ, 455, L147

Perlmutter, S., Aldering, G., Goldhaber, G., et al. 1999, ApJ, 517, 565

Phillips, M. M. 1993, ApJ, 413, L105

Rest, A., Stubbs, C., Becker, A. C., et al. 2005, ApJ, 634, 1103

Riess, A. G., Filippenko, A. V., Challis, P., et al. 1998, AJ, 116, 1009

Riess, A. G., Kirshner, R. P., Schmidt, B. P., et al. 1999, AJ, 11, 707

Riess, A. G., Macri, L., Casertano, S., et al. 2011, ApJ, 730, 119

Riess, A. G., Macri, L., Li, W., et al. 2009, ApJS, 183, 109

Riess, A. G., Press, W. H., \& Kirshner, R. P. 1996, ApJ, 473, 88

Riess, A. G., Strolger, L.-G., Casertano, S., et al. 2007, ApJ, 659, 98

Schechter, P. L., Mateo, M., \& Saha, A. 1993, PASP, 105, 1342

Schlegel, D. J., Finkbeiner, D. P., \& Davis, M. 1998, ApJ, 500, 525

Silverman, J. M., Ganeshalingam, M., Li, W., \& Filippenko, A. V. 2012, preprint (arXiv:1202.2130)

Silverman, J. M., Ganeshalingam, M., Li, W., et al. 2011, MNRAS, 410, 585

Smith, J. A., Tucker, D. L., Kent, S., et al. 2002, AJ, 123, 2121

Stanishev, V., Goobar, A., Benetti, S., et al. 2007, A\&A, 469, 645

Stritzinger, M., Hamuy, M., Suntzeff, N. B., et al. 2002, AJ, 124, 2100

Stritzinger, M., Phillips, M. M., Boldt, L. N., et al. 2011, AJ, 142, 156

Stubbs, C. W., \& Tonry, J. L. 2006, ApJ, 646, 1436

Sullivan, M., Conley, A., Howell, D. A., et al. 2010, MNRAS, 406, 782

Sullivan, M., Guy, J., Conley, A., et al. 2011, ApJ, 737, 102

Suntzeff, N. B. 2000, in AIP Conf. Proc. 522, Cosmic Explosions, ed. S. S. Holt \& W. W. Zhang (Melville, NY: AIP), 65

Suzuki, N., Rubin, D., Lidman, C., et al. 2012, ApJ, 746, 85

Taubenberger, S., Hachinger, S., Pignata, G., et al. 2008, MNRAS, 385, 75

Tody, D. 1993, in ASP Conf. Ser. 52, Astronomical Data Analysis Software and Systems II, ed. R. J. Hanisch, R. J. V. Brissenden, \& J. Barnes (San Francisco, CA: ASP), 173

Turnbull, S. J., Hudson, M. J., Feldman, H. A., et al. 2012, MNRAS, 420, 447

Wood-Vasey, W. M., Friedman, A. S., Bloom, J. S., et al. 2008, ApJ, 689, 377

Woodward, J. T., Brown, S. W., Lykke, K. R., et al. 2010, Proc. SPIE, 7737, 58

Zacharias, N., Finch, C., Girard, T., et al. 2010, AJ, 139, 2184 